\title{
MAXWELL'S EQUATIONS IN A PERIODIC STRUCTURE
}

\author{
XINFU CHEN AND AVNER FRIEDMAN
}

\begin{abstract}
Consider a diffraction of a beam of particles in $\mathbb{R}^{3}$ when the dielectric coefficient is a constant $\varepsilon_{1}$ above a surface $S$ and a constant $\varepsilon_{2}$ below a surface $S$, and the magnetic permeability is constant throughout $\mathbb{R}^{3}$. $S$ is assumed to be periodic in the $x_{1}$ direction and of the form $x_{1}=f_{1}(s)$, $x_{3}=f_{3}(s), x_{2}$ arbitrary. We prove that there exists a unique solution to the time-harmonic Maxwell equations in $\mathbb{R}^{3}$ having the form of refracted waves for $x_{3} \gg 1$ and of transmitted waves for $-x_{3} \gg 1$ if and only if there exists a unique solution to a certain system of two coupled Fredholm equations. Thus, in particular, for all the $\varepsilon$ 's, except for a discrete number, there exists a unique solution to the Maxwell equations.
\end{abstract}

\section{INTRODUCTION}

In this paper we consider the Maxwell equations for time harmonic solutions in the entire space $\mathbb{R}^{3}$, with piecewise constant dielectric coefficient having jump across a periodic surface. The magnetic permeability $\mu$ is assumed to be constant whereas the dielectric coefficient $\varepsilon$ is given by $\varepsilon=\varepsilon_{1}$ above a surface $S: x_{3}=f\left(x_{1}\right)$ and $\varepsilon=\varepsilon_{2}$ below the surface $S ; \varepsilon_{1}$ and $\varepsilon_{2}$ are different constants. If $S$ is a half-space $\left\{x_{3}=0\right\}$ then the solution $\vec{E}_{0}, \vec{H}_{0}$ can be computed explicitly. We assume in this paper that $S$ is periodic, i.e.,

$$
f\left(x_{1}+L\right)=f\left(x_{1}\right) \quad \forall x_{1} \in \mathbb{R}(L>0) .
$$

We wish to find a solution $\vec{E}, \vec{H}$ such that

$$
\begin{aligned}
& \vec{E}-\vec{E}_{0} \text { and } \vec{H}-\vec{H}_{0} \text { are superpositions of "transmitted" waves } \\
& \text { in }\left\{x_{3}<-A\right\} \text { and of "reflected" waves in }\left\{x_{3}>A\right\} \text { where } \\
& A>\max |f| .
\end{aligned}
$$

In $\S \S 1-7$ we assume that $f \in C^{2}$ and we reduce the solution of the Maxwell equations to a Fredholm system of four integral equations; in $\S 8$ we reduce it further to a Fredholm system of two integral equations. Thus for all but a discrete sequence of values of the physical parameters there exists a unique solution to the integral equations, yielding a solution of the Maxwell equations;

Received by the editors January 25, 1989.

1980 Mathematics Subject Classification (1985 Revision). Primary 78A10, 78A45; Secondary 35P25, 47A40.

Key words and phrases. Maxwell's equations, transmission, reflection, Fredholm equations.

This paper is partially supported by National Science Foundation Grant DMS-86-12880 
the solution satisfies (0.1). In $\S 9$ we prove that any solution of the Maxwell equations which satisfies $(0.1)$ is uniquely determined.

In $\S 10$ we generalize the previous results to the case where the curve $\hat{S} \equiv$ $S \cap\left\{x_{2}=0\right\}$ is not necessarily of the form $x_{3}=f\left(x_{1}\right)$ with $f \in C^{2}$; in fact $\hat{S}$ is assumed to be a piecewise $C^{2}$ curve, which is not necessarily an $x_{3}$-graph. In particular, the case where $f$ is a step-function is included; this case arises in the design of digital lenses (oral communication from Dr. Allen Cox at Honeywell).

Integral equations have been used by Benaldi [2] to solve the Maxwell equations; he used finite-elements schemes for computing the solutions of the integral equation. The Maxwell equations in periodic structure corresponding to arrays of antennas were studied by Nedelec and Starling [8]; Bellout and Friedman [1] studied the Schrödinger equation for a periodic potential, corresponding to quantum scattering by a slab with periodically varying potential energy.

For the specific problem dealt with in this paper, there is a numerical approach due to Gaylord and Moharam [5], which is based on approximating $f\left(x_{1}\right)$ by step-functions and using the "separation of variables" method for solving the approximating problems.

A good background on diffraction optics in grating material, especially from engineering and numerical points of view, can be found in a collection of articles edited by R. Petit [9].

\section{The Maxwell equations}

We denote points in $\mathbb{R}^{3}$ by $X=\left(x_{1}, x_{2}, x_{3}\right), Y=\left(y_{1}, y_{2}, y_{3}\right)$. Let $S$ be a surface in $\mathbb{R}^{3}$ given by $S$ : $x_{3}=f\left(x_{1}\right)$ where $f\left(x_{1}\right)$ is periodic of period $L$ :

$$
f\left(x_{1}+L\right)=f\left(x_{1}\right) \quad \forall x_{1}
$$

we also assume that $f \in C^{2}$.

Introduce the domains

$$
\begin{aligned}
& \Omega_{1}=\left\{X=\left(x_{1}, x_{2}, x_{3}\right) ; x_{1}>f\left(x_{3}\right)\right\}, \\
& \Omega_{2}=\left\{X=\left(x_{1}, x_{2}, x_{2}\right) ; x_{1}<f\left(x_{3}\right)\right\} .
\end{aligned}
$$

We assume that the magnetic permeability $\mu$ is constant throughout space whereas the dialectric coefficient $\varepsilon$ satisfies

$$
\varepsilon= \begin{cases}\varepsilon_{1} & \text { in } \Omega_{1}, \\ \varepsilon_{2} & \text { in } \Omega_{2},\end{cases}
$$

where $\varepsilon_{1}, \varepsilon_{2}$ are complex constants and $\varepsilon_{1} \neq \varepsilon_{2}$. Writing for $j=1,2$

$$
\varepsilon=\varepsilon^{\prime}+i \varepsilon^{\prime \prime}, \quad \varepsilon_{j}=\varepsilon_{j}^{\prime}+i \varepsilon_{j}^{\prime \prime},
$$

we further assume that

$$
\varepsilon_{j}^{\prime}>0, \quad \varepsilon_{j}^{\prime \prime} \geq 0
$$

the case $\varepsilon_{j}^{\prime \prime}>0$ accounts for absorption; see, for instance, [4, $\left.\S 82\right]$. 
Suppose a beam of particles is incident to the period surface $S$ from above and it is time periodic of period $2 \pi / \omega$. We wish to find the corresponding time harmonic solution

$$
\vec{E}(\vec{r}) e^{-i \omega t}, \vec{H}(\vec{r}) e^{-i \omega t} \quad\left(\vec{r}=X=\left(x_{1}, x_{2}, x_{3}\right)\right)
$$

of the Maxwell equations and, in particular, to investigate the asymptotic behavior of the solution as $\left|x_{3}\right| \rightarrow \infty$. Setting

$$
\vec{E}^{j}=\left.\vec{E}\right|_{\Omega_{j}}, \quad \vec{H}^{j}=\left.\vec{H}\right|_{\Omega_{j}},
$$

Maxwell's equations in each $\Omega_{j}$ are

$$
\begin{aligned}
& \nabla \times \vec{E}^{j}-\frac{i \omega \mu}{c} \vec{H}^{j}=0 \quad \text { in } \Omega_{j}, \\
& \nabla \times \vec{H}^{j}+\frac{i \omega \varepsilon}{c} \vec{E}^{j}=0 \quad \text { in } \Omega_{j},
\end{aligned}
$$

where $c$ is the speed of light. The weak form of Maxwell's equations in a neighborhood of $S$ reduces to the following jump relations:

$$
\begin{aligned}
\vec{n} \times\left(\vec{E}^{1}-\vec{E}^{2}\right)=0 & \text { on } S, \\
\vec{n} \times\left(\vec{H}^{1}-\vec{H}^{2}\right)=0 & \text { on } S, \\
\vec{n} \cdot\left(\varepsilon_{1} \vec{E}^{1}-\varepsilon_{2} \vec{E}^{2}\right)=0 & \text { on } S, \\
\vec{n} \cdot\left(\vec{H}^{1}-\vec{H}^{2}\right)=0 & \text { on } S,
\end{aligned}
$$

where $\vec{n}$ is the downward pointing unit normal to $S$.

The incident beam of particles coming from $\Omega_{1}$ can be represented by the solution

$$
\begin{gathered}
\vec{H}_{*}(\vec{r})=\left(0, h_{0}, 0\right), \quad \vec{E}_{*}(\vec{r})=\frac{c}{i \omega \varepsilon_{1}}\left(\frac{\partial h_{0}}{\partial x_{3}}, 0,-\frac{\partial h_{0}}{\partial x_{1}}\right), \\
h_{0}=e^{i\left(\alpha x_{1}-\beta x_{3}\right)},
\end{gathered}
$$

where

$$
\alpha^{2}+\beta^{2}=\frac{\omega^{2} \mu \varepsilon_{1}}{c^{2}}, \quad \alpha \text { real, } \operatorname{Im} \beta \geq 0
$$

In the special case $S=\left\{x_{3}=0\right\}$ the corresponding solution of the Maxwell equations (1.1) $-(1.6)$ is

$$
\begin{aligned}
\vec{H}_{0} & =(0, h, 0), \quad \vec{E}_{0}=\frac{c}{i \omega \varepsilon}\left(\frac{\partial h}{\partial x_{3}}, 0,-\frac{\partial h}{\partial x_{1}}\right), \\
h=h\left(x_{1}, x_{3}\right) & = \begin{cases}e^{i\left(\alpha x_{1}-\beta x_{3}\right)}+r(\alpha, \beta) e^{i\left(\alpha x_{1}+\beta x_{3}\right)} & \text { if } x_{3}>0, \\
t(\alpha, \beta) e^{i\left(\alpha x_{1}-\hat{\beta} x_{3}\right)} & \text { if } x_{3}<0,\end{cases}
\end{aligned}
$$

where

(1.10) $\hat{\beta}=\left\{\left(\alpha^{2}+\beta^{2}\right) n_{2}^{2}-\alpha^{2}\right\}^{1 / 2}, \quad \operatorname{Im} \hat{\beta} \geq 0, n_{2}=\left(\frac{\varepsilon_{2}}{\varepsilon_{1}}\right)^{1 / 2} \quad$ (Snell's law), 


$$
t(\alpha, \beta)=\frac{2 \beta n_{2}^{2}}{\beta n_{2}^{2}+\hat{\beta}} \quad(\text { transmission coefficient })
$$

$$
r(\alpha, \beta)=\frac{\beta n_{2}^{2}-\hat{\beta}}{\beta n_{2}^{2}+\hat{\beta}} \quad(\text { reflection coefficient }) .
$$

Notice that $\operatorname{Im} \beta>0$ and $\operatorname{Im} \hat{\beta}>0$ imply exponential decay away from $S$ (due to absorption).

We wish to find a solution to (1.1)-(1.6) for a general periodic surface $S$ such that $(0.1)$ holds; this last condition is precisely the condition (9.2) under which uniqueness is proved in $\S 9$.

We look for a solution satisfying:

$$
\begin{aligned}
& e^{-i \alpha x_{1}} \vec{E}(\vec{r}) \text { and } e^{-i \alpha x_{1}} \vec{H}(\vec{r}) \text { are periodic in } x_{1} \text { of period } L, \\
& \text { and are independent of } x_{2} .
\end{aligned}
$$

In the sequel we use the notation

$$
\vec{e}_{1}=(1,0,0), \quad \vec{e}_{2}=(0,1,0), \quad \vec{e}_{3}=(0,0,1) .
$$

Note that along $S$

$$
\vec{n}(X) \equiv \vec{n}\left(x_{1}\right)=\frac{f^{\prime}\left(x_{1}\right) \vec{e}_{1}-\vec{e}_{3}}{\left\{1+f^{\prime}\left(x_{1}\right)^{2}\right\}^{1 / 2}} .
$$

Throughout this paper we write

$$
\sigma\left(x_{1}\right)=\left\{1+f^{\prime}\left(x_{1}\right)^{2}\right\}^{1 / 2}
$$

We conclude this section by proving that equations $(1.5),(1.6)$ follow from (1.1)-(1.4).

Lemma 1.1. If $(\vec{E}, \vec{H})$ is a solution of $(1.1)-(1.4)$ then $(\vec{E}, \vec{H})$ satisfies also the equations (1.5), (1.6).

Proof. Take any bounded subdomain $F$ of $S$ with smooth boundary. By (1.1) (which is assumed to hold up to the boundary of $\Omega_{j}$ )

$$
\begin{aligned}
\int_{F} \vec{n} \cdot \frac{i \omega \mu}{c} \vec{H}^{j} d S & =\int_{F} \vec{n} \cdot\left(\nabla \times \vec{E}^{j}\right) d S=\int_{\partial F} \vec{E}^{j} \cdot\left(\vec{n} \times \vec{n}_{0}\right) d l \\
& =-\int_{\partial F} \vec{n}_{0} \cdot\left(\vec{n} \times \vec{E}^{j}\right) d l,
\end{aligned}
$$

where $\vec{n}_{0}$ is the outward unit normal to $\partial F$. Hence

$$
\int_{F} \frac{i \omega \mu}{c} \vec{n} \cdot\left(\vec{H}^{1}-\vec{H}^{2}\right) d S=-\int_{\partial F} \vec{n}_{0} \cdot\left(\vec{n} \times\left(\vec{E}^{1}-\vec{E}^{2}\right)\right) d l=0
$$

by (1.3). Since $F$ is arbitrary, $\vec{n} \cdot\left(\vec{H}^{1}-\vec{H}^{2}\right)=0$ on $S$. The proof of $(1.5)$ follows similarly from (1.2), (1.4). 


\section{INTEGRAL REPRESENTATION OF SOLUTIONS}

It is easily seen that $\vec{E}$ and $\vec{H}$ both satisfy, outside $S$, the equation

$$
\Delta v+k^{2} v=0, \quad \text { where } k=\frac{\omega}{c} \sqrt{\mu \varepsilon} ;
$$

we shall always choose $k$ such that

$$
\operatorname{Re} k>0, \quad \operatorname{Im} k \geq 0 .
$$

It is natural to expect an integral representation of $\vec{E}, \vec{H}$ by means of the fundamental solution $\Phi_{k}$ of (2.1):

$$
\Phi_{k}(X-Y)=\frac{e^{i k|X-Y|}}{4 \pi|X-Y|}
$$

$k=\frac{\omega}{c} \sqrt{\mu \varepsilon_{j}} \equiv k_{j}$ in $\Omega_{j}$.

Motivated by [7] we shall try to find a solution of the form

$$
\begin{aligned}
& \vec{E}(X)=\int_{S}\left\{\frac{i \omega \mu}{c} \vec{I}(Y) \Phi_{k}(X-Y)-\vec{J}(Y) \times \nabla_{Y} \Phi_{k}(X-Y)\right. \\
& \left.+\frac{c}{i \omega \varepsilon} \nabla_{X}\left[\vec{I}(Y) \cdot \nabla_{Y} \Phi_{k}(X-Y)\right]\right\} d S_{Y} \\
& \text { (2.4) } \vec{H}(X)=\int_{S}\left\{\frac{i \omega \varepsilon}{c} \vec{J}(Y) \Phi_{k}(X-Y)+\vec{I}(Y) \times \nabla_{Y} \Phi_{k}(X-Y)\right. \\
& \left.+\frac{c}{i \omega \mu} \nabla_{X}\left[\vec{J}(Y) \cdot \nabla_{Y} \Phi_{k}(X-Y)\right]\right\} d S_{Y} .
\end{aligned}
$$

Lemma 2.1. Suppose $\vec{I}(Y)$ and $\vec{J}(Y)$ are bounded by $O\left(|Y|^{-1-\delta}\right)$ as $|Y| \rightarrow \infty$, $\delta>0$ (so that the integrals in (2.3), (2.4) and their derivatives are all well defined). Then

$$
\begin{aligned}
& \nabla \times \vec{E}-\frac{i \omega \mu}{c} \vec{H}=0 \text { on } \mathbb{R}^{3} \backslash S, \\
& \nabla \times \vec{H}+\frac{i \omega \varepsilon}{c} \vec{E}=0 \quad \text { on } \mathbb{R}^{3} \backslash S .
\end{aligned}
$$

Proof. Since $\nabla_{X} \Phi_{k}=-\nabla_{Y} \Phi_{k}$, we have

$$
\vec{J} \times \nabla_{Y} \Phi_{k}=-\vec{J} \times \nabla_{X} \Phi_{k}=\nabla_{X} \times\left(\vec{J} \Phi_{k}\right) .
$$

It follows that

$$
\vec{E}=\frac{i \omega \mu}{c} \int \vec{I} \Phi_{k}-\nabla_{X} \times \int \vec{J} \Phi_{k}+\nabla_{X} \int \frac{c}{i \omega \varepsilon} \vec{I} \cdot \nabla_{Y} \Phi_{k} .
$$

Using the relations $\nabla \times \nabla=0$ and

$$
\nabla \times(\nabla \times \vec{g})=\nabla(\nabla \cdot \vec{g})-\Delta \vec{g},
$$


we deduce that

$$
\begin{aligned}
\nabla \times \vec{E}= & \frac{i \omega \mu}{c} \nabla_{X} \times \int \vec{I} \Phi_{k}-\nabla_{X} \times\left(\nabla_{X} \times \int \vec{J} \Phi_{k}\right) \\
& +\nabla_{X} \times\left[\nabla_{X} \int \frac{c}{i \omega \varepsilon} \vec{I} \cdot \nabla_{Y} \Phi_{k}\right] \\
= & \frac{i \omega \mu}{c} \int \vec{I} \times \nabla_{Y} \Phi_{k}-\nabla_{X}\left(\nabla_{X} \cdot \int \vec{J} \Phi_{k}\right)+\Delta_{X} \int \vec{J} \Phi_{k} \\
= & \frac{i \omega \mu}{c} \int\left\{\vec{I} \times \nabla_{Y} \Phi_{k}+\frac{c}{i \omega \mu} \nabla_{X}\left(\vec{J} \cdot \nabla_{Y} \Phi_{k}\right)+\frac{i \omega \varepsilon}{c} \vec{J} \Phi_{k}\right\} \\
= & \frac{i \omega \mu}{c} \vec{H}
\end{aligned}
$$

i.e., (2.5) holds. The proof of (2.6) is similar.

In the sequel we shall be interested only in $\vec{I}$ and $\vec{J}$ which are independent of $y_{2}$ and are such that

$$
e^{-i \alpha y_{1}} \vec{I}\left(y_{1}\right) \text { and } e^{-i \alpha y_{1}} \vec{J}\left(y_{1}\right) \text { are periodic in } y_{1} \text { of period } L \text {. }
$$

We then must show that the integrals in (2.3), (2.4) make sense. This is done in the next section.

\section{Periodic fundamental solution}

In the sequel we assume that

$$
k_{j}^{2} \neq\left(\frac{2 \pi n}{L}-\alpha\right)^{2} \text { for all } n=0, \pm 1, \pm 2, \ldots
$$

Notice that if $\operatorname{Im} \varepsilon_{j}>0$ then (3.1) is certainly satisfied.

Lemma 3.1. The following formula holds:

$$
\int_{-\infty}^{\infty} \Phi_{k}(X-Y) d y_{2}=\frac{i}{4} H_{0}^{(1)}(k|x-y|),
$$

where $x=\left(x_{1}, x_{3}\right), y=\left(y_{1}, y_{3}\right)$ and $H_{0}^{(1)}$ is a Hankel function.

Proof. Substituting $\zeta=\left(a^{2}+y^{2}\right)^{1 / 2} a^{-1 / 2}$, we get

$$
\int_{0}^{\infty} \frac{e^{i k\left(a^{2}+y^{2}\right)^{1 / 2}}}{\left(a^{2}+y^{2}\right)^{1 / 2}} d y=\int_{1}^{\infty} \frac{e^{i k a \zeta}}{\left(\zeta^{2}-1\right)^{1 / 2}} d \zeta=\frac{\pi i}{2} H^{(1)}(k a)
$$

in the last equality we used [6, p. 322, 387 \#4] for $\operatorname{Im} k \geq 0$, where $H_{0}^{(1)}$ is a Hankel function.

In the sequel we use the notation $x=\left(x_{1}, x_{3}\right), y=\left(y_{1}, y_{2}\right)$ and set

$$
\Psi_{k}(x-y)=\sum_{n=-\infty}^{\infty} \int_{-\infty}^{\infty} e^{-i \alpha\left(x_{1}-y_{1}+n L\right)} \Phi_{k}\left(X-Y+n L e_{1}\right) d y_{2} .
$$


For any complex number $k$ in $\mathbb{C}^{+}=\{\operatorname{Im} k>0\}$, set

$$
\begin{aligned}
\beta_{n}(k)= & \sqrt{\left|k^{2}-\left(\frac{2 \pi n}{L}-\alpha\right)^{2}\right|} e^{i \theta / 2} \\
& \text { if } k^{2}-\left(\frac{2 \pi n}{L}-\alpha\right)^{2}=\left|k^{2}-\left(\frac{2 \pi n}{L}-\alpha\right)^{2}\right| e^{i \theta}, 0 \leq \theta<2 \pi
\end{aligned}
$$

Since $k^{2} \neq(2 \pi n / L-\alpha)^{2}$ for $k \in \mathbb{C}^{+}, \beta_{n}(k)$ is a complex analytic function in $\mathbb{C}^{+}$; furthermore, it is continuous up to $\{\operatorname{Im} k=0\}$ and

$$
\beta_{n}(k)=\left\{\begin{array}{l}
\sqrt{k^{2}-\left(\frac{2 \pi n}{L}-\alpha\right)^{2}} \text { for } k \text { real, } k^{2}>\left(\frac{2 \pi n}{L}-\alpha\right)^{2}, \\
i \sqrt{\left(\frac{2 \pi n}{L}-\alpha\right)^{2}-k^{2}} \text { for } k \text { real, } k^{2}<\left(\frac{2 \pi n}{L}-\alpha\right)^{2} .
\end{array}\right.
$$

Theorem 3.2. For any $k \in \mathbb{C}^{+}$the following holds:

$$
\Psi_{k}(x-y)=\frac{i}{2 L} \sum_{n=-\infty}^{\infty} \frac{e^{i \beta_{n}(k)\left|x_{3}-y_{3}\right|} e^{-i(2 \pi n / L)\left(x_{1}-y_{1}\right)}}{\beta_{n}(k)} .
$$

Notice that for $k>0$ the infinite series contains a finite number of oscillating terms, namely those with $(2 \pi n / L-\alpha)^{2}<k^{2}$; the remaining terms are exponentially decaying as $\left|x_{3}-y_{3}\right| \rightarrow \infty$, and so is their sum. On the other hand, if $k \in \mathbb{C}^{+}$then all the terms in (3.6) are exponentially decaying as $\left|x_{3}-y_{3}\right| \rightarrow \infty$. Proof. It suffices to establish (3.6) for $y=0$. Set

$$
\begin{aligned}
& f\left(s, y, x_{3}\right)=e^{-i \alpha s} \frac{e^{i k \sqrt{s^{2}+y^{2}+x_{3}^{2}}}}{\sqrt{s^{2}+y^{2}+x_{3}^{2}}}, \\
& F\left(t, y, x_{3}\right)=\int_{-\infty}^{\infty} e^{i t s} f\left(s, y, x_{3}\right) d s .
\end{aligned}
$$

By the Poisson summation formula [3, p. 52]

$$
\sum_{n=-\infty}^{\infty} f\left(x_{1}+n L, y, x_{3}\right)=\frac{1}{L} \sum_{n=-\infty}^{\infty} F\left(\frac{2 \pi n}{L}, y, x_{3}\right) e^{-i 2 \pi n x_{1} / L} .
$$

Consequently

$$
\Psi_{k}(x)=\sum_{n=-\infty}^{\infty} \frac{1}{4 \pi L}\left(\int_{-\infty}^{\infty} F\left(\frac{2 \pi n}{L}, y, x_{3}\right) d y\right) e^{-i 2 \pi n x_{1} / L} .
$$

By Bellout-Friedman [1], each integral in the last sum is equal to

$$
\begin{aligned}
& 4 \int_{0}^{\infty} \int_{0}^{\infty} \cos \left[\left(\frac{2 \pi n}{L}-\alpha\right) s\right] \frac{e^{i k \sqrt{s^{2}+y^{2}+x_{3}^{2}}}}{\sqrt{s^{2}+y^{2}+x_{3}^{2}}} d s d y \\
& \quad=2 \pi \int_{0}^{\infty} \frac{r e^{i k \sqrt{r^{2}+x_{3}^{2}}}}{\sqrt{r^{2}+x_{3}^{2}}} J_{0}\left(\left|\frac{2 \pi n}{L}-\alpha\right| r\right) d r \equiv g_{n}(k) .
\end{aligned}
$$


Since

$$
J_{0}(z) \sim \sqrt{\frac{2}{\pi z}} \sin z \quad \text { as } z \rightarrow \infty,
$$

we easily deduce that the function $g_{n}(k)$ is complex analytic in $k$ for $k \in \mathbb{C}^{+}$, continuous up to $\operatorname{Im} k=0$. It was proved by Bellout and Friedman [1] that the boundary values of $g_{n}(k)$ on $\operatorname{Im} k=0$ are given by

$$
g_{n}(k)=2 \pi i \frac{e^{i \beta_{n}(k)\left|x_{3}\right|}}{\beta_{n}(k)} \text { for } k>0 .
$$

Since both functions

$$
g_{n}(k) \text { and } 2 \pi i \frac{e^{i \beta_{n}(k)\left|x_{3}\right|}}{\beta_{n}(k)}
$$

are complex analytic in $\mathbb{C}^{+}$with the same boundary values on $\operatorname{Im} k=0$, $k>0$, it follows, by applying unique continuation to their difference, that they must coincide in $\mathbb{C}^{+}$. Thus (3.8) holds for all complex $k, \operatorname{Im} k \geq 0$, and using this in (3.7) the assertion (3.6) follows.

Remark 3.1. Recall, by [6, p. 951, $8.405 \# 1]$, that

$$
H_{0}^{(1)}(k a)=J_{0}(k a)+i N_{0}(k a)
$$

and, as $z \rightarrow 0$,

$$
\begin{aligned}
& J_{0}(z)=1+O(z) \quad[6, \text { p. } 959,8.440] \\
& \pi N_{0}(z)=2 J_{0}(z) \log z+O(z) \quad[6, \text { p. } 960,8.444] .
\end{aligned}
$$

It follows that

$$
H_{0}^{(1)}(z)=\frac{2 i}{\pi} \log z+O(1) \quad \text { as } z \rightarrow 0 .
$$

Setting

$$
\Phi_{k, 0}(z)=\int_{-\infty}^{\infty} \Phi_{k}(X) d x_{2},
$$

we conclude from Lemma 3.1 that

$$
\Phi_{k, 0}(x)=\frac{1}{2 \pi} \log \frac{1}{|x|}+O(1) \quad(|x| \rightarrow 0) .
$$

Remark 3.2. Set

$$
\begin{gathered}
\Phi_{k, n}(x)=\int_{-\infty}^{\infty} \Phi_{k}\left(x_{1}+n, x_{2}, x_{3}\right) d x_{2}, \\
g_{N}=\sum_{\substack{n=-N \\
n \neq 0}}^{N} \Phi_{k, n} .
\end{gathered}
$$

Then

$$
\Delta g_{N}+k^{2} g_{N}=0 \text { for all } x,-L / 2 \leq x_{1} \leq L / 2,
$$


and $g_{N}$ is uniformly convergent for $|x|=R,(\forall 0<R<L / 2)$ since the same is true of $g_{N}+\Phi_{k, 0}$ (by Theorem 3.2). Hence, by elliptic estimates, $g_{N}$ is uniformly convergent also in $\{|x|<R\}$. It follows that

$$
\Psi_{k}(x-y)-e^{-i \alpha\left(x_{1}-y_{1}\right)} \Phi_{k, 0}(x-y)
$$

is a smooth function also as $x-y \rightarrow 0$ and then, by Remark 3.1,

$$
\Psi_{k}(x-y)-\frac{1}{2 \pi} \log \frac{1}{|x-y|} \text { is smooth } \forall x, y .
$$

The above argument is due to Nedelec and Starling [8].

Remark 3.3. If we use Lemma 3.1 and apply the Poisson summation formula to $f(s)=e^{-\alpha s} H_{0}^{(1)}\left(k \sqrt{s^{2}+x_{3}^{2}}\right)$, and then compare the resulting expression for $\Psi_{k}(x)$ with that derived in Theorem 3.2, we obtain a formula for the Fourier transform of $H_{0}^{(1)}\left(k \sqrt{s^{2}+a^{2}}\right)$ for $\operatorname{Im} k \geq 0$; this formula is already known in case $\operatorname{Im} k=0$ (see [6, p. 736]).

\section{INTEGRAL REPRESENTATION FOR THE PERIODIC CASE}

Set

$$
\vec{E}_{\alpha}(X)=e^{-i \alpha x_{1}} \vec{E}(X), \quad \vec{H}_{\alpha}(X)=e^{-i \alpha x_{1}} \vec{H}(X) .
$$

We can assume that $\vec{I}$ and $\vec{J}$ depend only on $y_{1}$, and set

$$
\vec{I}_{\alpha}\left(y_{1}\right)=e^{-i \alpha y_{1}} \vec{I}\left(y_{1}\right), \quad \vec{J}_{\alpha}\left(y_{1}\right)=e^{-i \alpha y_{1}} \vec{J}\left(y_{1}\right) .
$$

Multiplying both sides of (2.3) by $e^{-i \alpha x_{1}}$ and setting

$$
\Phi_{k, \alpha}(X-Y)=e^{-i \alpha\left(x_{1}-y_{1}\right)} \Phi_{k}(X-Y),
$$

we get, after some easy manipulations,

$$
\begin{aligned}
\vec{E}_{\alpha}(X)= & \int_{S}\left\{\frac{i \omega \mu}{c} \vec{I}_{\alpha} \Phi_{k, \alpha}(X-Y)-\vec{J}_{\alpha} \times \nabla_{Y} \Phi_{k, \alpha}(X-Y)\right. \\
& \left.\quad+\frac{c}{i \omega \varepsilon} \nabla_{X}\left[\vec{I}_{\alpha}\left(y_{1}\right) \cdot \nabla_{Y} \Phi_{k, \alpha}(X-Y)\right]\right\} \\
& +i \alpha \int_{S}\left(\vec{J}_{\alpha} \times \vec{e}_{1}\right) \Phi_{k, \alpha}(X-Y) \\
& +\frac{i c \alpha}{i \omega \varepsilon} \int\left[\vec{I}_{\alpha} \cdot \nabla_{Y} \Phi_{k, \alpha}(X-Y) \vec{e}_{1}+\left(\vec{I}_{\alpha} \cdot \vec{e}_{1}\right) \nabla_{Y} \Phi_{k, \alpha}(X-Y)\right] \\
& -\frac{c(i \alpha)^{2}}{i \omega \varepsilon} \vec{e}_{1} \int_{S} \vec{I}_{\alpha} \cdot \vec{e}_{1} \Phi_{k, \alpha}(X-Y) .
\end{aligned}
$$

If we integrate with respect to $y_{2},-\infty<y_{2}<\infty$, we obtain on the righthand side of (4.4), the same expressions but with $\Phi_{\alpha}(X-Y)$ replaced by

$$
\widetilde{\Phi}_{k, \alpha}(x-y) \equiv \int_{-\infty}^{\infty} \Phi_{k, \alpha}\left(x_{1}-y_{1}, x_{2}-y_{2}, x_{3}-y_{3}\right) d y_{2} .
$$


Indeed, we first replace each $\nabla_{Y} \Phi_{\alpha}$ by $-\nabla_{X} \Phi_{\alpha}$, then perform the integration with respect to $y_{2}$, and finally replace back the $-\nabla_{X} \widetilde{\Phi}_{\alpha}$ by $\nabla_{Y} \widetilde{\Phi}_{\alpha}$. Assuming that

$$
\vec{I}_{\alpha}\left(y_{1}\right), \vec{J}_{\alpha}\left(y_{1}\right) \text { are } L \text {-periodic in } y_{1},
$$

we can then rewrite (4.4) in the form

$$
\begin{aligned}
\vec{E}_{\alpha}(X)= & \int_{0}^{L}\left\{\frac{i \omega \mu}{c} \vec{I}_{\alpha} \Psi_{k}(x-\tilde{y})-\vec{J}_{\alpha} \times \nabla_{Y} \Psi_{k}(x-\tilde{y})\right. \\
& \left.\quad+\frac{c}{i \omega \varepsilon} \nabla_{X}\left[\vec{I}_{\alpha}\left(y_{1}\right) \cdot \nabla_{Y} \Psi_{k}(x-\tilde{y})\right]\right\} \sigma \\
& +i \alpha \int_{0}^{L}\left(\vec{J}_{\alpha} \times \vec{e}_{1}\right) \Psi_{k}(x-\tilde{y}) \sigma \\
& +\frac{c \alpha}{\omega \varepsilon} \int_{0}^{L}\left[\vec{I}_{\alpha} \cdot \nabla_{Y} \Psi_{k}(x-\tilde{y}) \vec{e}_{1}+\left(\vec{I}_{\alpha} \cdot \vec{e}_{1}\right) \nabla_{Y} \Psi_{k}(x-\tilde{y})\right] \sigma \\
& -\frac{i c \alpha^{2}}{\omega \varepsilon} \vec{e}_{1} \int_{0}^{L}\left(\vec{I}_{\alpha} \cdot \vec{e}_{1}\right) \Psi_{k}(x-\tilde{y}) \sigma d y_{1},
\end{aligned}
$$

where $\sigma=\sigma\left(y_{1}\right)$ is defined as in (1.16), and $\tilde{y}=\left(y_{1}, f\left(y_{1}\right)\right)$.

Using the notation

$$
\nabla_{x}=\left(\frac{\partial}{\partial x_{1}}, 0, \frac{\partial}{\partial x_{3}}\right), \quad \nabla_{y}=\left(\frac{\partial}{\partial y_{1}}, 0, \frac{\partial}{\partial y_{3}}\right)
$$

omitting the index $\alpha$ in $\vec{I}_{\alpha}, \vec{J}_{\alpha}$, and noting that the right-hand side of (4.6) is independent of $x_{2}$, we can rewrite (4.6) in the form

$$
\begin{aligned}
\vec{E}_{\alpha}(x)= & \int_{0}^{L}\left\{\frac{i \omega \mu}{c} \vec{I} \Psi_{k}(x-\tilde{y})-\vec{J} \times \nabla_{y} \Psi_{k}(x-\tilde{y})\right. \\
& \left.\quad+\frac{c}{i \omega \varepsilon} \nabla_{x}\left[\vec{I}\left(y_{1}\right) \cdot \nabla_{y} \Psi_{k}(x-\tilde{y})\right]\right\} \sigma\left(y_{1}\right) d y_{1} \\
& +i \alpha \int_{0}^{L}\left(\vec{J} \times \vec{e}_{1}\right) \Psi_{k}(x-\tilde{y}) \sigma\left(y_{1}\right) d y_{1} \\
& +\frac{c \alpha}{\omega \varepsilon} \int_{0}^{L}\left[\vec{I} \cdot \nabla_{y} \Psi_{k}(x-\tilde{y}) \vec{e}_{1}+I_{1} \nabla_{y} \Psi_{k}(x-\tilde{y})\right] \sigma\left(y_{1}\right) d y_{1} \\
& -\frac{i c \alpha^{2}}{\omega \varepsilon} \vec{e}_{1} \int_{0}^{L} I_{1} \Psi_{k}(x-\tilde{y}) \sigma\left(y_{1}\right) d y
\end{aligned}
$$

where $\vec{I}=\vec{I}\left(y_{1}\right)=\left(I_{1}, I_{2}, I_{3}\right), \vec{J}=\vec{J}(y)=\left(J_{1}, J_{2}, J_{3}\right)$; here we wrote $\vec{E}_{\alpha}(x)=\vec{E}_{\alpha}(X)$. 
Similarly, from (2.4) we obtain

$$
\begin{aligned}
\vec{H}_{\alpha}(x)= & \int_{0}^{L}\left\{\frac{i \omega \varepsilon}{c} \vec{J} \Psi_{k}(x-\tilde{y})+\vec{I} \times \nabla_{y} \Psi_{k}(x-\tilde{y})\right. \\
& \left.\quad+\frac{c}{i \omega \mu} \nabla_{x}\left[\vec{J}\left(y_{1}\right) \cdot \nabla_{y} \Psi_{k}(x-\tilde{y})\right]\right\} \sigma\left(y_{1}\right) d y_{1} \\
& -i \alpha \int_{0}^{L}\left(\vec{I} \times \vec{e}_{1}\right) \Psi_{k}(x-\tilde{y}) \sigma\left(y_{1}\right) d y_{1} \\
& +\frac{c \alpha}{\omega \mu} \int_{0}^{L}\left[\vec{J} \cdot \nabla_{y} \Psi_{k}(x-\tilde{y}) \vec{e}_{1}+J_{1} \nabla_{y} \Psi_{k}(x-\tilde{y})\right] \sigma\left(y_{1}\right) d y_{1} \\
& -\frac{i c \alpha^{2}}{\omega \mu} \vec{e}_{1} \int_{0}^{L} J_{1} \Psi_{k}(x-\tilde{y}) \sigma\left(y_{1}\right) d y_{1} .
\end{aligned}
$$

Lemma 4.1. For any functions $\vec{I}\left(y_{1}\right), \vec{J}\left(y_{1}\right) \quad\left(0<y_{1}<L\right)$ the functions $\vec{E}, \vec{H}$ defined by (4.1), (4.7) and (4.8) satisfy the Maxwell equations (2.5), (2.6); further

$$
\vec{E}_{\alpha}\left(x_{1}+L, x_{3}\right)=\vec{E}_{\alpha}\left(x_{1}, x_{3}\right), \quad \vec{H}_{\alpha}\left(x_{1}+L, x_{3}\right)=\vec{H}_{\alpha}\left(x_{1}, x_{2}\right) .
$$

Proof. To prove (2.5), (2.6) we can proceed by appealing to Lemma 2.1 and rigorously establishing the passage from (2.3), (2.4) to (4.7), (4.8). Alternately (and more simply) we can establish (2.5), (2.6) directly, using the method of proof of Lemma 2.1. Finally, the validity of (4.9) is obvious.

We shall henceforth refer to $S$ as either the surface $\left\{x_{3}=f\left(x_{1}\right)\right\}$ in $\mathbb{R}^{3}$ or the curve $\left\{x_{3}=f\left(x_{1}\right)\right\}$ in $\mathbb{R}^{2}$. We denote points on the curve $S$ by

$$
\tilde{x}=\left(x_{1}, f\left(x_{1}\right)\right), \quad \tilde{y}=\left(y_{1}, f\left(y_{1}\right)\right) .
$$

Lemma 4.2. If $\vec{I}\left(y_{1}\right)$ is L-periodic in $y_{1}$ and if $\vec{I} \cdot \vec{n}=0$ on $S$, then, for any $z \neq 0$,

$$
\int_{0}^{L} \vec{I}\left(y_{1}\right) \cdot\left(\nabla_{y} \Psi_{k}\left(\tilde{x}_{z}-\tilde{y}\right)\right) \sigma\left(y_{1}\right) d y_{1}=-\int_{0}^{L} \rho(\vec{I}) \Psi_{k}\left(\tilde{x}_{z}-\tilde{y}\right) d y_{1}
$$

where $\tilde{x}_{z}=\left(x_{1}+z, f\left(x_{1}\right)\right)$, and

$$
\rho(\vec{I})=\frac{d}{d y_{1}}\left(I_{1}\left(y_{1}\right) \sigma\left(y_{1}\right)\right), \quad \vec{I}=\left(I_{1}, I_{2}, I_{3}\right) .
$$

Proof. Since $\vec{I} \cdot \vec{n}=0$,

$$
I_{3}=I_{1} f^{\prime}
$$

Hence

$$
\begin{aligned}
\int_{0}^{L} \sigma \vec{I} \cdot \nabla_{y} \Psi_{k}\left(\tilde{x}_{z}-\tilde{y}\right)=\int_{0}^{L} \sigma \vec{I} \cdot\left(\frac{\partial \Psi_{k}}{\partial y_{1}} \vec{e}_{1}+\frac{\partial \Psi_{k}}{\partial y_{3}} \vec{e}_{3}\right) \\
\quad=\int_{0}^{L}\left(\sigma I_{1} \frac{\partial \Psi_{k}}{\partial y_{1}}+\sigma I_{3} \frac{\partial \Psi_{k}}{\partial y_{3}}\right)=\int_{0}^{L} \sigma I_{1} \frac{d}{d y_{1}} \Psi_{k}=-\int_{0}^{L} \frac{d}{d y_{1}}\left(\sigma I_{1}\right) \Psi_{k}
\end{aligned}
$$


the boundary terms disappear since $\sigma I_{1} \Psi_{k}$ is periodic in $y_{1}$. This completes the proof.

Using Lemma 4.2 we can rewrite the formulas (4.7), (4.8) in a more convenient way, by getting rid of some gradients $\nabla_{y}$. The new representation is

$$
\begin{aligned}
\vec{E}_{\alpha}(x)= & \int_{0}^{L}\left\{\frac{i \omega \mu}{c} \sigma \vec{I} \Psi_{k}(x-\tilde{y})-\sigma \vec{J} \times \nabla_{y} \Psi_{k}(x-\tilde{y})\right. \\
& \left.+\frac{c}{i \omega \varepsilon} \rho(\vec{I}) \nabla_{y} \Psi_{k}(x-\tilde{y})\right\} d y_{1} \\
& +i \alpha \int_{0}^{L}\left(\vec{J} \times \vec{e}_{1}\right) \Psi_{k}(x-\tilde{y}) \sigma\left(y_{1}\right) d y_{1}-\frac{c \alpha}{\omega \varepsilon} \int_{0}^{L} \rho(\vec{I}) \Psi_{k}(x-\tilde{y}) \vec{e}_{1} d y_{1} \\
& +\frac{c \alpha}{\omega \varepsilon} \int_{0}^{L} \sigma\left(y_{1}\right) I_{1} \nabla_{y} \Psi_{k}(x-\tilde{y}) d y_{1}-\frac{i c \alpha^{2}}{\omega \varepsilon} \vec{e}_{1} \int_{0}^{L} \vec{I}_{1} \Psi_{k}(x-\tilde{y}) \sigma\left(y_{1}\right) d y_{1},
\end{aligned}
$$

$$
\begin{aligned}
\vec{H}_{\alpha}(x)= & \int_{0}^{L}\left\{\frac{i \omega \varepsilon}{c} \sigma \vec{J} \Psi_{k}(x-\tilde{y})+\sigma \vec{I} \times \nabla_{y} \Psi_{k}(x-\tilde{y})\right. \\
& \left.\quad+\frac{c}{i \omega \mu} \rho(\vec{J}) \nabla_{y} \Psi_{k}(x-\tilde{y})\right\} d y_{1} \\
& -i \alpha \int_{0}^{L} K\left(\vec{I} \times \vec{e}_{1}\right) \Psi_{k}(x-\tilde{y}) \sigma\left(y_{1}\right) d y_{1}-\frac{c \alpha}{\omega \mu} \int_{0}^{L} \rho(\vec{J}) \Psi_{k}(x-\tilde{y}) e_{1} d y_{1} \\
& +\frac{c \alpha}{\omega \mu} \int_{0}^{L} \sigma\left(y_{1}\right) J_{1} \nabla_{y} \Psi_{k}(x-\tilde{y}) d y_{1} \\
& -\frac{i c \alpha^{2}}{\omega \mu} \vec{e}_{1} \int_{0}^{L} \vec{J}_{1} \Psi_{k}(x-\tilde{y}) \sigma\left(y_{1}\right) d y_{1} .
\end{aligned}
$$

\section{Auxiliary estimates}

Let

$$
\Psi^{*}(x)=\frac{1}{2 \pi} \sum_{n=-\infty}^{\infty} \log \frac{n L}{\left|x+n L \vec{e}_{1}\right|} .
$$

The series is convergent in the sense of principal value, i.e.,

$$
\Psi^{*}(x)=\frac{1}{2 \pi} \lim _{N \rightarrow \infty} \sum_{n=-N}^{N} \log \frac{n L}{\left|x+n L \vec{e}_{1}\right|} ;
$$

further

$$
\nabla \Psi^{*}(x)=-\frac{1}{2 \pi} \sum_{n=-\infty}^{\infty} \frac{\left(x_{1}+n L, x_{3}\right)}{\left(x_{1}+n L\right)^{2}+x_{3}^{2}}
$$

where the convergence is again in the sense of principal value, i.e.,

$$
\nabla \Psi^{*}(x)=-\frac{1}{2 \pi} \lim _{N \rightarrow \infty} \sum_{n=-N}^{N} \frac{\left(x_{1}+n L, x_{3}\right)}{\left(x_{1}+n L\right)^{2}+x_{3}^{2}}
$$


the convergence is uniform in $x$ in every compact set which does not contain integers.

Set

$$
\Psi_{0}^{*}(x)=\frac{1}{2 \pi} \log \frac{1}{|x|} .
$$

Lemma 5.1. For any $z \neq 0$, write $\tilde{x}_{z}=\tilde{x}+z \vec{e}_{3}$ and let

$$
\vec{R}_{k, z}(\tilde{x}, \tilde{y}) \equiv \nabla_{y} \Psi_{k}\left(\tilde{x}_{z}-\tilde{y}\right)-\nabla_{y} \Psi^{*}\left(x_{1}-y_{1}, z+f^{\prime}\left(x_{1}\right)\left(x_{1}-y_{1}\right)\right),
$$

where $\nabla_{y} \Psi_{k}=-\nabla \Psi_{k}$. Then

$$
\left|\vec{R}_{k, z}(\tilde{x}, \tilde{y})\right| \leq C,
$$

where $C$ is a constant independent of $\tilde{x}, \tilde{y}, z$.

Proof. By (3.9) and (5.2) it suffices to show that

$$
\left|\nabla_{y} \Psi_{0}^{*}\left(\tilde{x}_{z}-\tilde{y}\right)-\nabla_{y} \Psi_{0}^{*}\left(x_{1}-y_{1}, z+f^{\prime}\left(x_{1}\right)\left(x_{1}-y_{1}\right)\right)\right| \leq C,
$$

i.e.,

$$
\begin{aligned}
& K \equiv \mid \frac{\left(x_{1}-y_{1}, z+f\left(x_{1}\right)-f\left(y_{1}\right)\right)}{\left(x_{1}-y_{1}\right)^{2}+\left(z+f\left(x_{1}\right)-f\left(y_{1}\right)\right)^{2}} \\
& \quad-\frac{\left(x_{1}-y_{1}, z+f^{\prime}\left(x_{1}\right)\left(x_{1}-y_{1}\right)\right)}{\left(x_{1}-y_{1}\right)^{2}+\left(z+f^{\prime}\left(x_{1}\right)\left(x_{1}-y_{1}\right)\right)^{2}} \mid \leq C .
\end{aligned}
$$

The difference of the numerators is $\left(0, f\left(x_{1}\right)-f\left(y_{1}\right)-f^{\prime}\left(x_{1}\right)\left(x_{1}-y_{1}\right)\right)$, which is $O\left(\left(x_{1}-y_{1}\right)^{2}\right)$. Hence

$$
\begin{aligned}
\left.K \leq C_{0}+\left(\left|x_{1}-y_{1}\right|+|z|\right)\right)\{2 \mid & \left|z\left[f^{\prime}\left(x_{1}\right)\left(x_{1}-y_{1}\right)-\left(f\left(x_{1}\right)-f\left(y_{1}\right)\right)\right]\right| \\
+ & \left.\left|f^{\prime}\left(x_{1}\right)^{2}\left(x_{1}-y_{1}\right)^{2}-\left(f\left(x_{1}\right)-f\left(y_{1}\right)\right)^{2}\right|\right\} / N
\end{aligned}
$$

where $N$ is the product of the denominators, or

$$
K \leq C_{0}+C_{1}\left(\left|x_{1}-y_{1}\right|+|z|\right)\left[|z|\left(x_{1}-y_{1}\right)^{2}+\left|x_{1}-y_{1}\right|^{3}\right] / N .
$$

If $|z|>\left(1+C_{2}\right)\left|x_{1}-y_{1}\right|$ where $C_{2}=\max \left|f^{\prime}\right|$, then $N \geq c_{0}|z|^{4} \quad\left(c_{0}>0\right)$ and consequently $K \leq C$. On the other hand if $|z|<\left(1+C_{2}\right)\left|x_{1}-y_{1}\right|$ then

$$
K \leq C_{0}+C_{3}\left|x_{1}-y_{1}\right|^{4} / N \leq C_{0}+C_{4} .
$$

Thus $K$ is bounded in both cases, independently of $z, \tilde{x}, \tilde{y}$.

Lemma 5.2. For all $z \neq 0$,

$$
\int_{0}^{L} \nabla \Psi^{*}\left(x_{1}, z+a x_{1}\right) d x_{1}=-\frac{1}{2} \frac{\vec{e}_{3}-a \vec{e}_{1}}{1+a^{2}} \operatorname{sgn} z .
$$

Proof. Notice that $\nabla \Psi^{*}$ is defined as in (5.2). Therefore, the left-hand side of (5.6) is equal to the integral

$$
\int_{-\infty}^{\infty} \nabla \Psi_{0}^{*}\left(x_{1}, z+a x_{1}\right) d x_{1}
$$


taken in the p.v. (principal value) sense:

$$
\lim _{N \rightarrow \infty} \int_{-N}^{N} \nabla \Psi_{0}^{*}\left(x_{1}, z+a x_{1}\right) d x_{1} .
$$

To compute the integral in (5.7) we write it in the form

$$
\begin{aligned}
-\frac{1}{2 \pi} \int_{-\infty}^{\infty} & \frac{x_{1} \vec{e}_{1}+\left(z+a x_{1}\right) \vec{e}_{3}}{x_{1}^{2}+\left(z+a x_{1}\right)^{2}} d x_{1} \\
= & -\frac{1}{2 \pi} \int_{-\infty}^{\infty} \frac{\left(x_{1}+a z /\left(1+a^{2}\right)\right)\left(\vec{e}_{1}+a \vec{e}_{3}\right)}{\left(1+a^{2}\right)\left(x_{1}+a z /\left(1+a^{2}\right)\right)^{2}+z^{2} /\left(1+a^{2}\right)} \\
& -\frac{1}{2 \pi} \int_{-\infty}^{\infty} \frac{\left(z /\left(1+a^{2}\right)\right)\left(\vec{e}_{3}-a \vec{e}_{1}\right)}{\left(1+a^{2}\right)\left(x_{1}+a z /\left(1+a^{2}\right)\right)^{2}+z^{2} /\left(1+a^{2}\right)} d x_{1} .
\end{aligned}
$$

The first integral on the right-hand side (taken in the p.v. sense) is equal to zero since the integrand is an odd function in $x_{1}+a z /\left(1+a^{2}\right)$. Since further

$$
\frac{1}{2 \pi} \int_{-\infty}^{\infty} \frac{\varepsilon}{\left(1+a^{2}\right)\left(\xi_{1}^{2}+\varepsilon^{2}\right)} d \xi_{1}=\frac{1}{2} \frac{\operatorname{sgn} \varepsilon}{1+a^{2}}
$$

the assertion of the lemma follows.

Lemma 5.3. Let $u\left(x_{1}\right)$ be an L-periodic function, locally in $L^{2}$. Then

$$
\begin{aligned}
& \lim _{\substack{z \rightarrow 0 \\
z \neq 0}} \int_{0}^{L}\left(u\left(y_{1}\right)-u\left(x_{1}\right)\right) \nabla_{y} \Psi^{*}\left(x_{1}-y_{1}, z+a\left(x_{1}-y_{1}\right)\right) d y_{1} \\
& \quad=\int_{0}^{L}\left(u\left(y_{1}\right)-u\left(x_{1}\right)\right) \nabla_{y} \Psi^{*}\left(x_{1}-y_{1}, a\left(x_{1}-y_{1}\right)\right) d y_{1}=\frac{1}{2} \frac{\vec{e}_{1}+a \vec{e}_{3}}{1+a^{2}} H(u),
\end{aligned}
$$

where $\nabla_{y} \Psi^{*}$ means $-\operatorname{grad} \Psi^{*}$, and

$$
\begin{aligned}
H u & =(H u)\left(x_{1}\right)=\text { p.v. } \frac{1}{\pi} \int_{-\infty}^{\infty} \frac{u\left(y_{1}\right)}{x_{1}-y_{1}} d y_{1} \\
& =\lim _{\substack{\varepsilon \rightarrow 0 \\
N \rightarrow \infty}}\left\{\frac{1}{\pi} \int_{-N}^{x_{1}-\varepsilon} \frac{u\left(y_{1}\right)}{x_{1}-y_{1}} d y_{1}+\frac{1}{\pi} \int_{x_{1}+\varepsilon}^{N} \frac{u\left(y_{1}\right)}{x_{1}-y_{1}} d y_{1}\right\}
\end{aligned}
$$

is the Hilbert transform.

Proof. From (5.2) we see that (5.8) is equivalent to

$$
\begin{aligned}
& \lim _{\substack{z \rightarrow 0 \\
z \neq 0}} \int_{-\infty}^{\infty}\left(u\left(y_{1}\right)-u\left(x_{1}\right)\right) \nabla_{y} \Psi_{0}^{*}\left(x_{1}-y_{1}, z+a\left(x_{1}-y_{1}\right)\right) d y_{1} \\
& \quad=\int_{-\infty}^{\infty}\left(u\left(y_{1}\right)-u\left(x_{1}\right)\right) \nabla_{y} \Psi_{0}^{*}\left(x_{1}-y_{1}, a\left(x_{1}-y_{1}\right)\right) d y_{1} \\
& \quad=\frac{1}{2} \frac{\vec{e}_{1}+a \vec{e}_{3}}{1+a^{2}} H u
\end{aligned}
$$

the integrals are taken in the p.v. sense, $\lim _{N \rightarrow \infty} \int_{-N}^{N}$. 
Consider first the integral of the middle term of (5.10); it is equal to

$$
\begin{aligned}
\frac{1}{2 \pi} \int_{-\infty}^{\infty}\left(u\left(y_{1}\right)-u\left(x_{1}\right)\right) \frac{\left(x_{1}-y_{1}\right)\left(\vec{e}_{1}+a \vec{e}_{3}\right)}{\left(1+a^{2}\right)\left(x_{1}-y_{1}\right)^{2}} d y_{1} \\
\quad=-\frac{\vec{e}_{1}+a \vec{e}_{3}}{2\left(1+a^{2}\right)} \frac{1}{\pi} \int_{-\infty}^{\infty} \frac{u\left(y_{1}\right)-u\left(x_{1}\right)}{\left|x_{1}-y_{1}\right|} \operatorname{sgn}\left(x_{1}-y_{1}\right) d y_{1} \\
=-\frac{\vec{e}_{1}+a \vec{e}_{3}}{2\left(1+a^{2}\right)} \frac{1}{\pi} \int_{-\infty}^{\infty} \frac{u\left(y_{1}\right)}{x_{1}-y_{1}} d y_{1}=\frac{\vec{e}_{1}+a \vec{e}_{3}}{2\left(1+a^{2}\right)} H(u),
\end{aligned}
$$

since

$$
\text { p.v. } \int_{-\infty}^{\infty} \frac{u\left(x_{1}\right)}{x_{1}-y_{1}} d y_{1}=0 .
$$

Thus the second equality in (5.10) follows. The first equality in (5.10) follows by similar considerations.

\section{THE JUMP RELATIONS}

Fix a point $\tilde{x}$. For any $z>0$, the point $x=\tilde{x}+z \vec{e}_{3}$ belongs to $\Omega_{1}$. We shall evaluate $\lim _{z \rightarrow 0} \vec{E}_{\alpha}\left(\tilde{x}+z e_{3}\right)$, where $\vec{E}_{\alpha}$ is defined by (4.12).

Notice that all the integrals which do not involve $\nabla_{y} \Psi_{k}$ are well defined also at $z=0$ and are continuous up to $z \geq 0$. Thus the sum of the terms in $\vec{E}_{\alpha}$ which may produce some discontinuity is

$$
\begin{aligned}
& -\int_{0}^{L} \sigma\left(y_{1}\right) \vec{J}\left(y_{1}\right) \times \nabla_{y} \Psi_{k}(x-\tilde{y}) d y_{1}+\int_{0}^{L} \frac{c}{i \omega \varepsilon} \rho(\vec{I}) \nabla_{y} \Psi_{k}(x-\tilde{y}) d y_{1} \\
& +\frac{c \alpha}{\omega \varepsilon} \int_{0}^{L} \sigma\left(y_{1}\right) I_{1} \nabla_{y} \Psi_{k}(x-\tilde{y}) d y_{1} .
\end{aligned}
$$

Write the first term in (6.1) in the form

$$
\begin{aligned}
& -\int_{0}^{L} \sigma\left(y_{1}\right) \vec{J}\left(y_{1}\right) \times \vec{R}_{k, z}(\tilde{x}, \tilde{y}) d y_{1} \\
& \quad-\int_{0}^{L}\left[\sigma\left(y_{1}\right) \vec{J}\left(y_{1}\right)-\sigma\left(x_{1}\right) \vec{J}\left(x_{1}\right)\right] \times \nabla_{y} \Psi^{*}\left(x_{1}-y_{1}, z+a\left(x_{1}-y_{1}\right)\right) d y_{1} \\
& \quad-\sigma\left(x_{1}\right) \vec{J}\left(x_{1}\right) \times \int_{0}^{L} \nabla_{y} \Psi^{*}\left(x_{1}-y_{1}, z+a\left(x_{1}-y_{1}\right)\right) d y_{1} \quad\left(a=f^{\prime}\left(x_{1}\right)\right) .
\end{aligned}
$$

Then, as $z \downarrow 0$ we get, by Lemmas 5.1-5.3,

$$
\begin{aligned}
& -\int_{0}^{L} \sigma\left(y_{1}\right) \vec{J}\left(y_{1}\right) \times \vec{R}_{k}\left(x_{1}, y_{1}\right) d y_{1}-\frac{1}{2} H(\sigma \vec{J}) \times \frac{\vec{e}_{1}+a \vec{e}_{3}}{1+a^{2}} \\
& -\frac{1}{2} \sigma \vec{J} \times \frac{\vec{e}_{3}-a \vec{e}_{1}}{1+a^{2}}
\end{aligned}
$$

where

$$
\vec{R}_{k}\left(x_{1}, y_{1}\right)=\nabla_{y} \Psi_{k}(\tilde{x}-\tilde{y})-\nabla_{y} \Psi^{*}\left(x_{1}-y_{1}, f^{\prime}\left(x_{1}\right)\left(x_{1}-y_{1}\right)\right)
$$


Recalling (1.15) and introducing the unit tangent

$$
\vec{\tau}=\frac{\vec{e}_{1}+f^{\prime}\left(x_{1}\right) \vec{e}_{3}}{\left\{1+f^{\prime}\left(x_{1}\right)^{2}\right\}^{1 / 2}}
$$

to $S$, we can write with $(6.2)$ in the form

$$
-\int_{0}^{L} \sigma\left(y_{1}\right) \vec{J}\left(y_{1}\right) \times \vec{R}_{k}\left(x_{1}, y_{1}\right) d y_{1}-\frac{H(\sigma \vec{J})}{2 \sigma} \times \vec{\tau}+\frac{1}{2} \vec{J} \times \vec{n} .
$$

If $z<0, z \rightarrow 0$ then we obtain the same limit except that $\frac{1}{2} \vec{J} \times \vec{n}$ is replaced by $-\frac{1}{2} \vec{J} \times \vec{n}$.

Similarly, for the second term in (6.1) we get, as $z \downarrow 0$,

$$
\frac{c}{i \omega \varepsilon} \int_{0}^{L} \rho(\vec{I}) \vec{R}_{k}\left(x_{1}, y_{1}\right) d y_{1}+\frac{c}{i \omega \varepsilon} \frac{H(\rho(\vec{I}))}{2 \sigma} \vec{\tau}-\frac{1}{2} \frac{c}{i \omega \varepsilon} \frac{\rho(\cdot \vec{I})}{\sigma} \vec{n},
$$

and for the third term we get the limit

$$
\frac{c \alpha}{\omega \varepsilon} \int_{0}^{L} \sigma\left(y_{1}\right) I_{1}\left(y_{1}\right) \vec{R}_{k}\left(x_{1}, y_{1}\right) d y_{1}+\frac{c \alpha}{\omega \varepsilon} \frac{H\left(\sigma I_{1}\right)}{2 \sigma} \vec{\tau}-\frac{1}{2} \frac{c \alpha}{\omega \varepsilon} I_{1} \vec{n} .
$$

If $z<0, z \rightarrow 0$ we get the same results, but the last term in (6.6) and in (6.7) have the reverse sign.

Setting $\Psi_{j}=\Psi_{k_{j}}, \vec{R}_{j}=\vec{R}_{k_{j}}$ and

$$
\vec{E}_{\alpha}^{j}(\tilde{x})=\lim _{\substack{x \rightarrow \tilde{x}_{j} \\ x \in \Omega_{j}}} \vec{E}_{\alpha}(X)
$$

we conclude that

$$
\begin{aligned}
\vec{E}_{\alpha}^{j}(\tilde{x})= & \int_{0}^{L}\left\{\frac{i \omega \mu}{c} \sigma \vec{I} \Psi_{j}-\sigma \vec{J} \times \vec{R}_{j}+\frac{c}{i \omega \varepsilon_{j}} \rho(\vec{I}) \vec{R}_{j}\right\} \\
& +i \alpha \int_{0}^{L}\left(\vec{J} \times \vec{e}_{1}\right) \sigma \Psi_{j}-\frac{c \alpha}{\omega \varepsilon_{j}} \int_{0}^{L} \rho(\vec{I}) \Psi_{j} \vec{e}_{1}+\frac{c \alpha}{\omega \varepsilon_{j}} \int_{0}^{L} \sigma I_{1} \vec{R}_{j} \\
& -\frac{i c \alpha^{2}}{\omega \varepsilon_{j}} \vec{e}_{1} \int_{0}^{L} I_{1} \sigma \Psi_{j}-\frac{1}{2 \sigma} H(\sigma \vec{J}) \times \vec{\tau}+\frac{c}{i \omega \varepsilon_{j}} \frac{H(\rho(\vec{I}))}{2 \sigma} \vec{\tau} \\
& +\frac{c \alpha}{\omega \varepsilon_{j}} \frac{H\left(\sigma I_{1}\right)}{2 \sigma} \vec{\tau} \pm \frac{1}{2} \vec{J} \times \vec{n} \mp \frac{c}{i \omega \varepsilon_{j}} \frac{\rho(\vec{I})}{2 \sigma} \vec{n} \mp \frac{1}{2} \frac{c \alpha}{\omega \varepsilon_{j}} I_{1} \vec{n},
\end{aligned}
$$

where "+" is for $j=1$ and " - " for $j=2$; here $\Psi_{j}=\Psi_{j}(\tilde{x}-\tilde{y})$ and $\vec{R}_{j}=\vec{R}_{j}\left(x_{1}, y_{1}\right)$.

Similarly, setting

$$
\vec{H}_{\alpha}^{j}(\tilde{x})=\lim _{\substack{x \rightarrow x^{x} \\ x \in \Omega_{j}}} \vec{H}_{\alpha}(x)
$$


we get

$$
\begin{aligned}
\vec{H}_{\alpha}^{j}(\tilde{x})= & \int_{0}^{L}\left\{\frac{i \omega \varepsilon_{j} \sigma}{c} \vec{J} \Psi_{j}+\sigma \vec{I} \times \vec{R}_{j}+\frac{c}{i \omega \mu} \rho(\vec{J}) \vec{R}_{j}\right\} \\
& -i \alpha \int_{0}^{L}\left(\vec{I} \times \vec{e}_{1}\right) \sigma \Psi_{j}-\frac{c \alpha}{\omega \mu} \int_{0}^{L} \rho(\vec{J}) \Psi_{j} \vec{e}_{1}+\frac{c \alpha}{\omega \mu} \int_{0}^{L} \sigma J_{1} \vec{R}_{j} \\
& -\frac{i c \alpha^{2}}{\omega \mu} \vec{e}_{1} \int_{0}^{L} J_{1} \sigma \Psi_{j}+\frac{1}{2 \sigma} H(\sigma \vec{I}) \times \vec{\tau}+\frac{c}{i \omega \mu} \frac{H(\rho(\vec{J}))}{2 \sigma} \vec{\tau} \\
& +\frac{c \alpha}{\omega \mu} \frac{H\left(\sigma J_{1}\right)}{2 \sigma} \vec{\tau} \mp \frac{1}{2} \vec{I} \times \vec{n} \mp \frac{c}{i \omega \mu} \frac{\rho(\vec{J})}{2 \sigma} \vec{n} \mp \frac{1}{2} \frac{c \alpha}{\omega \mu} J_{1} \vec{n} .
\end{aligned}
$$

We summarize

Lemma 6.1. If $\vec{I} \cdot \vec{n}=0, \vec{J} \cdot \vec{n}=0$ along $S$, then the $\vec{E}_{\alpha}(x), \vec{H}_{\alpha}(x)$ defined by (4.7), (4.8) are uniformly continuous in each $\bar{\Omega}_{j}$ and their limits on $\partial \Omega_{j}$ are given by (6.8), (6.9) respectively.

\section{REDUCTION TO INTEGRAL EQUATIONS}

Definition 7.1. We denote by $\left(\vec{E}_{f}, \vec{H}_{f}\right)$ the vector field defined by (1.9)-(1.12), where $x_{3}>0$ and $x_{3}<0$, in (1.9), are replaced by $x_{3}>f\left(x_{1}\right)$ and $x_{3}<f\left(x_{1}\right)$ respectively.

We shall try to solve $(1.1)-(1.4)$ in the form

$$
(\vec{E}, \vec{H})=\left(\vec{E}_{f}, \vec{H}_{f}\right)+\left(\vec{E}_{\alpha}, \vec{H}_{\alpha}\right) e^{i \alpha x_{1}}
$$

where $\left(\vec{E}_{\alpha}, \vec{H}_{\alpha}\right)$ are defined by (4.7), (4.8) for some $L$-periodic surface fields $\vec{I}, \vec{J}$ (i.e., $\vec{I} \cdot \vec{n}=0, \vec{J} \cdot \vec{n}=0$ ). A solution of the form (7.1) will incorporate the condition (1.13), and as we shall see later on, the condition (0.1) will also be satisfied.

Set

$$
\begin{array}{r}
T_{1}(\vec{I}, \vec{J})=\int_{0}^{L}\left\{\frac{i \omega \mu}{c} \sigma \vec{I}\left(\Psi_{1}-\Psi_{2}\right)-\sigma \vec{J} \times\left(\vec{R}_{1}-\vec{R}_{2}\right)+\frac{c}{i \omega} \rho(\vec{I})\left(\frac{\vec{R}_{1}}{\varepsilon_{1}}-\frac{\vec{R}_{2}}{\varepsilon_{2}}\right)\right. \\
+i \alpha\left(\vec{J} \times \vec{e}_{1}\right) \sigma\left(\Psi_{1}-\Psi_{2}\right)-\frac{c \alpha}{\omega} \rho(\vec{I}) \vec{e}_{1}\left(\frac{\Psi_{1}}{\varepsilon_{1}}-\frac{\Psi_{2}}{\varepsilon_{2}}\right) \\
\left.+\frac{c \alpha}{\omega} \sigma I_{1}\left(\frac{\vec{R}_{1}}{\varepsilon_{1}}-\frac{\vec{R}_{2}}{\varepsilon_{2}}\right)-\frac{i c \alpha^{2}}{\omega} I_{1} \vec{e}_{1} \sigma\left(\frac{\Psi_{1}}{\varepsilon_{1}}-\frac{\Psi_{2}}{\varepsilon_{2}}\right)\right\}
\end{array}
$$

$$
\begin{array}{r}
T_{2}(\vec{I}, \vec{J})=\int_{0}^{L}\left\{\frac{i \omega}{c} \sigma \vec{J}\left(\varepsilon_{1} \Psi_{1}-\varepsilon_{2} \Psi_{2}\right)+\sigma \vec{I} \times\left(\vec{R}_{1}-\vec{R}_{2}\right)+\frac{c}{i \omega \mu} \rho(\vec{J})\left(\vec{R}_{1}-\vec{R}_{2}\right)\right. \\
-i \alpha\left(\vec{I} \times \vec{e}_{1}\right) \sigma\left(\Psi_{1}-\Psi_{2}\right)-\frac{c \alpha}{\omega \mu} \rho(\vec{J})\left(\Psi_{1}-\Psi_{2}\right) \vec{e}_{1} \\
\left.+\frac{c \alpha}{\omega \mu} \sigma J_{1}\left(\vec{R}_{1}-\vec{R}_{2}\right)-\frac{i c \alpha^{2}}{\omega \mu} \sigma J_{1} \vec{e}_{1}\left(\Psi_{1}-\Psi_{2}\right)\right\} .
\end{array}
$$


Lemma 7.1. $(\vec{E}, \vec{H})$ is a solution of (1.1)-(1.4) if and only if $\vec{I}, \vec{J}$ satisfy the following conditions:

$$
\begin{gathered}
\vec{n} \times\left(\vec{H}_{0}^{1}-\vec{H}_{0}^{2}\right) e^{-i \alpha x_{1}}-\vec{I}+\vec{n} \times T_{2}(\vec{I}, \vec{J})=0, \\
\vec{n} \times\left(\vec{E}_{0}^{1}-\vec{E}_{0}^{2}\right) e^{-\alpha x_{1}}+\vec{J}+\frac{\varepsilon_{1}-\varepsilon_{2}}{\varepsilon_{1} \varepsilon_{2}} \frac{c \vec{e}_{2}}{2 i \sigma \omega}\left[H(\rho(\vec{I}))+i \alpha H\left(\sigma I_{1}\right)\right] \\
+\vec{n} \times T_{1}(\vec{I}, \vec{J})=0 .
\end{gathered}
$$

Here and in the sequel, $\vec{H}_{0}^{j}$ and $\vec{E}_{0}^{j}$ stand for $\vec{E}_{f}$ and $\vec{H}_{f}$ in $\boldsymbol{\Omega}_{j}$.

Proof. If $\vec{E}, \vec{H}$ satisfy (1.3), (1.4) then (7.4), (7.5) follow using (6.8), (6.9) and noting that $\vec{n} \times \vec{\tau}=-\vec{e}_{2}, \vec{n} \times(\vec{J} \times \vec{n})=\vec{J}, \vec{n} \times(\vec{I} \times \vec{n})=\vec{I}$. Conversely, if $\vec{I}, \vec{J}$ satisfy (7.4), (7.5) then clearly $\vec{I} \cdot \vec{n}=0, \vec{J} \cdot \vec{n}=0$ and therefore if $\vec{E}_{\alpha}, \vec{H}_{\alpha}$ are defined by $(4.7),(4.8)$ then they satisfy $(6.8),(6.9)$. It follows that (7.4), (7.5) imply (1.3), (1.4).

Definition 7.2. Introduce the space

(7.6) $X=\left\{g\left(x_{1}\right) ; g\left(x_{1}\right)\right.$ is $L$-periodic continuous function on $\mathbb{R}^{1}$

$$
\text { with } \left.\|g\|=\max _{-L / 2 \leq x \leq L / 2}\left|g\left(x_{1}\right)\right|\right\} \text {. }
$$

Consider the operator

$$
T: g \rightarrow \int_{0}^{L} G\left(x_{1}, y_{1}\right) g\left(y_{1}\right) d y_{1},
$$

where $G\left(x_{1}, y_{1}\right)$ is continuous for all $\left(x_{1}, y_{1}\right), x_{1} \neq y_{1}$, and $\left|G\left(x_{1}, y_{1}\right)\right| \leq$ $C\left|x_{1}-y_{1}\right|^{-1+\varepsilon} \quad(\varepsilon>0)$. Then $T$ is a compact operator from $X$ into $X$.

We need to look more carefully at $T_{1}$ and $T_{2}$. By integration by parts

$$
\begin{gathered}
\int_{0}^{L} \rho(\vec{J})\left(\vec{R}_{1}-\vec{R}_{2}\right)=-\int_{0}^{L} \sigma J_{1} \frac{d}{d y_{1}}\left(\vec{R}_{1}-\vec{R}_{2}\right), \\
\int_{0}^{L} \rho(\vec{J})\left(\Psi_{1}-\Psi_{2}\right) \vec{e}_{1}=-\int_{0}^{L} \sigma J_{1} \vec{e}_{1} \frac{d}{d y_{1}}\left(\Psi_{1}-\Psi_{2}\right) .
\end{gathered}
$$

Using this in $T_{2}$ we find that

$$
T_{2}=\int_{0}^{L} \frac{i \omega}{c} \sigma\left(\varepsilon_{1}-\varepsilon_{2}\right) \vec{J} \Psi_{1}+\hat{T}_{2},
$$


where

$$
\begin{aligned}
& \hat{T}_{2}(\vec{I}, \vec{J})=\int_{0}^{L}\left\{\frac{i \omega}{c} \sigma \varepsilon_{2} \vec{J}\left(\Psi_{1}-\Psi_{2}\right)+\sigma \vec{I} \times\left(\vec{R}_{1}-\vec{R}_{2}\right)\right.-\frac{c}{i \omega \mu} \sigma J_{1} \frac{d}{d y_{1}}\left(\vec{R}_{1}-\vec{R}_{2}\right) \\
&-i \alpha\left(\vec{I} \times \vec{e}_{1}\right) \sigma\left(\Psi_{1}-\Psi_{2}\right)+\frac{c \alpha}{\omega \mu} \sigma J_{1} \frac{d}{d y_{1}}\left(\Psi_{1}-\Psi_{2}\right) \vec{e}_{1} \\
&\left.\quad+\frac{c \alpha}{\omega \mu} \alpha J_{1}\left(\vec{R}_{1}-\vec{R}_{2}\right)-\frac{i c \alpha^{2}}{\omega \mu} \sigma J_{1} \vec{e}_{1}\left(\Psi_{1}-\Psi_{2}\right)\right\} \\
& \equiv \int_{0}^{L} G_{2}\left(x_{1}, y_{1}\right)(\vec{I}, \vec{J}) ;
\end{aligned}
$$

$G_{2}$ is a $3 \times 6$ matrix and $(\vec{I}, \vec{J})$ is a column vector with components $I_{j}, J_{j}$. Since $\Psi_{1}-\Psi_{2}$ and $\vec{R}_{1}-\vec{R}_{2}$ are smooth functions,

$$
\left|\nabla G_{2}\right| \leq C \quad\left(\nabla=\left(\frac{\partial}{\partial x_{1}}, \frac{\partial}{\partial y_{1}}\right)\right) .
$$

In particular, $\hat{T}_{2}$ is a compact operator from $X$ into $X$ (more precisely, from $X^{6}$ into $X^{3}$ ).

Similarly we can write

$$
T_{1}=\left(\frac{1}{\varepsilon_{1}}-\frac{1}{\varepsilon_{2}}\right) \frac{c}{i \omega} \int_{0}^{L} \rho(\vec{I})\left(\vec{R}_{1}-i \alpha \Psi_{1} \vec{e}_{1}\right)+\hat{T}_{1},
$$

where

$$
\begin{gathered}
\hat{T}_{1}(\vec{I}, \vec{J})=\int_{0}^{L}\left\{\frac{i \omega \mu}{c} \sigma \vec{I}\left(\Psi_{1}-\Psi_{2}\right)-\sigma \vec{J} \times\left(\vec{R}_{1}-\vec{R}_{2}\right)\right. \\
\quad-\frac{c}{i \omega \varepsilon_{2}} \sigma I_{1} \frac{d}{d y_{1}}\left(\vec{R}_{1}-\vec{R}_{2}\right) \\
+i \alpha\left(\vec{J} \times \vec{e}_{1}\right) \sigma\left(\Psi_{1}-\Psi_{2}\right)+\frac{c \alpha}{\omega \varepsilon_{2}} \sigma I_{1}\left(\Psi_{1}-\Psi_{2}\right) \vec{e}_{1} \\
\left.\quad+\frac{c \alpha}{\omega} \sigma I_{1}\left(\frac{\vec{R}_{1}}{\varepsilon_{1}}-\frac{\vec{R}_{2}}{\varepsilon_{2}}\right)-\frac{i c \alpha^{2}}{\omega} I_{1} \vec{e}_{1} \sigma\left(\frac{\Psi_{1}}{\varepsilon_{1}}-\frac{\Psi_{2}}{\varepsilon_{2}}\right)\right\} \\
\equiv \int_{0}^{L} G_{1}\left(x_{1}, y_{1}\right)(\vec{I}, \vec{J})
\end{gathered}
$$

and $G_{1}$ is a $3 \times 6$ matrix,

$$
\left|G_{1}\left(x_{1}, y_{1}\right)\right| \leq C \log \frac{1}{\left|x_{1}-y_{1}\right|}+C_{1}
$$

$G_{1}\left(x_{1}, y_{1}\right)$ is continuous in $x_{1}, y_{1}$ for all $x_{1} \neq y_{1}$. It follows that $\hat{T}_{1}$ is a compact operator from $X$ into $X$. 
If we substitute (7.8), (7.11) into (7.4), (7.5), we get

$$
\begin{aligned}
\vec{n} \times & \left(\vec{H}_{0}^{1}-\vec{H}_{0}^{2}\right) e^{-i \alpha x_{1}}-\vec{I} \\
& +\frac{i \omega}{\varepsilon}\left(\varepsilon_{1}-\varepsilon_{2}\right) \vec{n} \times \int_{0}^{L} \sigma \vec{J} \Psi_{1}+\vec{n} \times \hat{T}_{2}(\vec{I}, \vec{J})=0,
\end{aligned}
$$

$$
\begin{aligned}
n \times\left(\vec{E}_{0}^{1}-\vec{E}_{0}^{2}\right) e^{-i \alpha x_{1}}+\vec{J}+\frac{\varepsilon_{1}-\varepsilon_{2}}{\varepsilon_{1} \varepsilon_{2}} \frac{c \vec{e}_{2}}{2 i \sigma \omega}\left[H(\rho(\vec{I}))+i \alpha H\left(\sigma I_{1}\right)\right] \\
+\frac{\varepsilon_{2}-\varepsilon_{1}}{\varepsilon_{1} \varepsilon_{2}} \frac{c}{i \omega} \vec{n} \times \int_{0}^{L} \rho(\vec{I})\left(\vec{R}_{1}-i \alpha \Psi_{1} \varepsilon_{1}\right)+\vec{n} \times \hat{T}_{1}=0 .
\end{aligned}
$$

Lemma 7.2. If $\vec{K}=\vec{n} \times \int_{0}^{L} \sigma \vec{J} \Psi_{j}$ and $\vec{J} \cdot \vec{n}=0$ then

$$
\rho(\vec{K})=\frac{d}{d x_{1}}\left(K_{1} \sigma\right)=-\frac{1}{2} H\left(\sigma J_{2}\right)-\int_{0}^{L} \sigma J_{2}\left(R_{j}^{1}+f^{\prime}\left(x_{1}\right) R_{j}^{3}\right) d y_{1},
$$

where $\vec{R}_{j}=\left(R_{j}^{1}, R_{j}^{2}, R_{j}^{3}\right)$.

Proof. Notice that

$$
\vec{J}=J_{1}\left(\vec{e}_{1}+f^{\prime}\left(x_{1}\right) \vec{e}_{3}\right)+J_{2} \vec{e}_{2} .
$$

We have

$$
K_{1}=\vec{K} \cdot \vec{e}_{1}=-\left(\vec{n} \times \vec{e}_{1}\right) \cdot \int_{0}^{L} \sigma \vec{J} \Psi_{j}=\frac{1}{\sigma} \int_{0}^{L} \sigma J_{2} \Psi_{j} .
$$

Let $x_{z}=\tilde{x}+z \vec{e}_{3}=\left(x_{1}, z+f\left(x_{1}\right)\right) \quad(z \neq 0)$ and consider

$$
K_{1}(z)=\frac{1}{\sigma\left(x_{1}\right)} \int_{0}^{L} \sigma\left(y_{1}\right) J_{2}\left(y_{1}\right) \Psi_{j}\left(x_{z}-\tilde{y}\right) d y_{1} .
$$

Then

$$
\begin{aligned}
\frac{d}{d x_{1}}\left(K_{1}(z) \sigma\right)= & \int_{0}^{L} \sigma\left(y_{1}\right) J_{2}\left(y_{1}\right)\left(\frac{\partial \Psi_{j}}{\partial x_{1}}+f^{\prime}\left(x_{1}\right) \frac{\partial \Psi_{j}}{\partial x_{3}}\right)\left(x_{z}-\tilde{y}\right) d y_{1} \\
= & \sigma\left(x_{1}\right) J_{2}\left(x_{1}\right) \int_{0}^{L}\left(\frac{\partial \Psi^{*}}{\partial x_{1}}+f^{\prime}\left(x_{1}\right) \frac{\partial \Psi^{*}}{\partial x_{3}}\right) \\
& \left(x_{1}-y_{1}, z+f^{\prime}\left(x_{1}\right)\left(x_{1}-y_{1}\right)\right) d y_{1} \\
& +\int_{0}^{L}\left[\sigma\left(y_{1}\right) J_{2}\left(y_{1}\right)-\sigma\left(x_{1}\right) J_{2}\left(x_{1}\right)\right]\left(\frac{\partial \Psi^{*}}{\partial x_{1}}+f^{\prime}\left(x_{1}\right) \frac{\partial \Psi^{*}}{\partial x_{3}}\right) \\
& \left(x_{1}-y_{1}, z+f^{\prime}\left(x_{1}\right)\left(x_{1}-y_{1}\right)\right) d y_{1} \\
& -\int_{0}^{L} \sigma\left(y_{1}\right) J_{2}\left(y_{1}\right)\left[R_{k_{j}, z}^{1}(\tilde{x}, \tilde{y})+f^{\prime}\left(x_{1}\right) R_{k_{j}, z}^{3}(\tilde{x}, \tilde{y})\right] d y_{1},
\end{aligned}
$$

where $R_{k_{j}, z}$ is defined as in (5.4).

Using calculations as in Lemma 5.2, we find that the first integral on the right-hand side is equal to zero. Similarly, by the calculations in Lemma 5.3, 
the second integral on the right-hand side is continuous in $z$ (up to $z=0$ ) and is equal to $-\frac{1}{2} H\left(\sigma J_{2}\right)$ when $z=0$.

Since the last integral in (7.17) is also continuous in $z$, up to $z=0$, we conclude that (7.16) holds.

Let us now substitute $\vec{I}$ from (7.14) into those expressions in (7.15) which involve $\rho(\vec{I})$, noting that by Lemma 7.2 ,

$$
\begin{aligned}
\rho(\vec{I})= & \rho\left(\vec{n} \times\left(\vec{H}_{0}^{1}-\vec{H}_{0}^{2}\right) e^{i \alpha x_{1}}\right)+\rho\left(\vec{n} \times \hat{T}_{2}(\vec{I}, \vec{J})\right) \\
& -\frac{i \omega}{c}\left(\varepsilon_{1}-\varepsilon_{2}\right)\left\{\frac{1}{2} H\left(\sigma J_{2}\right)+\int_{0}^{L} \sigma J_{2}\left(R_{1}^{1}+f^{\prime}\left(x_{1}\right) R_{3}^{1}\right)\right\} .
\end{aligned}
$$

Since $H^{2} u=H^{2}\left(u \chi_{[-L, L]}\right)+H^{2}\left(u \chi_{[-L, L]^{c}}\right)$ and $H^{2} v=-v$ for any $v \in$ $L^{2}\left(\mathbb{R}^{1}\right)$, we obtain

$$
\begin{gathered}
\vec{n} \times\left(\vec{E}_{0}^{1}-\vec{E}_{0}^{2}\right) e^{-\alpha x_{1}}+\vec{J}+\frac{\left(\varepsilon_{1}-\varepsilon_{2}\right)^{2}}{4 \varepsilon_{1} \varepsilon_{2}}\left(J_{2} e_{2}+e_{2} H^{2}\left(\chi_{[-L, L]} J_{2}\right)\right) \\
+\frac{\varepsilon_{1}-\varepsilon_{2}}{\varepsilon_{1} \varepsilon_{2}} \frac{c \vec{e}_{2}}{2 i \sigma \omega}\left\{H \left[\rho\left(\vec{n} \times\left(\vec{H}_{0}^{1}-\vec{H}_{0}^{2}\right) e^{-i \alpha x_{1}}\right)+\rho\left(\hat{n} \times \hat{T}_{2}(\vec{I}, \vec{J})\right)\right.\right. \\
\left.\quad-\frac{i \omega}{c}\left(\varepsilon_{1}-\varepsilon_{2}\right) \int_{0}^{L} \sigma J_{2}\left(R_{1}^{1}+f^{\prime}\left(x_{1}\right) R_{3}^{1}\right)\right] \\
\left.+i \alpha H\left(\sigma I_{1}\right)\right\} \\
+\frac{\varepsilon_{2}-\varepsilon_{1}}{\varepsilon_{1} \varepsilon_{2}} \frac{c}{i \omega} \vec{n} \int_{0}^{L}\left\{\rho\left(\vec{n} \times\left(\vec{H}_{0}^{1}-\vec{H}_{0}^{2}\right) e^{-i \alpha x_{1}}\right)+\rho\left(\vec{n} \times \hat{T}_{2}(\vec{I}, \vec{J})\right)\right. \\
\left.-\frac{i \omega}{c}\left(\varepsilon_{1}-\varepsilon_{2}\right)\left[\frac{1}{2} H\left(\sigma J_{2}\right)+\int_{0}^{L} \sigma J_{2}\left(R_{1}^{1}+f^{\prime}\left(x_{1}\right) R_{3}^{1}\right)\right]\right\} \\
\cdot\left(\vec{R}_{1}-i \alpha \Psi_{1} \vec{e}_{1}\right)+\vec{n} \times \hat{T}_{1}=0 .
\end{gathered}
$$

In view of $(7.10)$,

$$
\hat{T}_{3} \equiv \rho\left(\vec{n} \times \hat{T}_{2}\right) \text { is a compact integral operator. }
$$

Also, from (7.14)

(7.21) $\vec{I}=\vec{n} \times\left(\vec{H}_{0}^{1}-\vec{H}_{0}^{2}\right) e^{-i \alpha x_{1}}+\hat{T}_{4}(\vec{I}, \vec{J}), \quad \hat{T}_{4}$ a compact integral operator.

Using (7.21) in evaluating the term $i \alpha H\left(\sigma I_{1}\right)$ in (7.19), using also (7.20), and noting that

$$
\int H\left(\sigma J_{2}\right)\left(\vec{R}_{1}-\alpha \Psi_{1} \vec{e}_{1}\right)
$$

is a compact operator, we can rewrite (7.19) in the form

$$
\vec{J}+\frac{\left(\varepsilon_{1}-\varepsilon_{2}\right)^{2}}{4 \varepsilon_{1} \varepsilon_{2}} J_{2} \vec{e}_{2}=\vec{J}_{0}+\hat{T}_{5}(\vec{I}, \vec{J}),
$$


where $\vec{J}_{0}$ depends only on the vectors $\vec{E}_{0}^{1}-\vec{E}_{0}^{2}, \vec{H}_{0}^{1}-\vec{H}_{0}^{2}$, and it vanishes if both vectors vanish, and $\hat{T}_{5}(\vec{I}, \vec{J})$ is a compact integral operator.

If we finally denote

$$
J_{2}+\frac{\left(\varepsilon_{1}-\varepsilon_{2}\right)^{2}}{4 \varepsilon_{1} \varepsilon_{2}} \text { by } J_{2},
$$

we see that $(7.21),(7.22)$ reduce to

$$
\vec{I}=\vec{I}_{0}+\hat{T}_{4}(\vec{I}, \vec{J}), \quad \vec{J}=\vec{J}_{0}+\hat{T}_{5}(\vec{I}, \vec{J})
$$

with $\vec{J}_{0}$ as above, $\vec{I}_{0}=n \times\left(\vec{H}_{0}^{1}-\vec{H}_{0}^{2}\right) e^{-i \alpha x_{1}}$, and slightly modified $\hat{T}_{4}, \hat{T}_{5}$.

We have proved

Theorem 7.3. The system (7.4), (7.5) is equivalent to the system (7.23) where $\hat{T}_{4}, \hat{T}_{5}$ are compact linear integral operators in $X ;$ consequently the Fredholm alternative holds.

It follows that the system has a unique solution except for a countable number of values of the parameters $\varepsilon_{1}, \varepsilon_{2}, \mu$.

\section{A SIMPLIFIED INTEGRAL SYSTEM}

Recall that

$$
\begin{aligned}
& \vec{\tau}\left(x_{1}\right)=\frac{1}{\sigma\left(x_{1}\right)}\left(\vec{e}_{1}+f^{\prime}\left(x_{1}\right) \vec{e}_{3}\right), \\
& \vec{n}\left(x_{1}\right)=\frac{1}{\sigma\left(x_{1}\right)}\left(f^{\prime}\left(x_{1}\right) \vec{e}_{1}-\vec{e}_{3}\right)
\end{aligned}
$$

and $\left(\vec{e}_{2}, \vec{\tau}\left(x_{1}\right), \vec{n}\left(x_{1}\right)\right)$ is a moving orthonormal frame along $S$, with $\vec{\tau} \times \vec{n}=$ $\vec{e}_{2}$.

Let us write the possible solution $\vec{I}, \vec{J}$ of (7.23) in the form

$$
\begin{aligned}
& \vec{I}\left(x_{1}\right)=I_{\tau}\left(x_{1}\right) \vec{\tau}\left(x_{1}\right)+I_{2}\left(x_{1}\right) \vec{e}_{2}, \\
& \vec{J}\left(x_{1}\right)=J_{\tau}\left(x_{1}\right) \vec{\tau}\left(x_{1}\right)+J_{2}\left(x_{1}\right) \vec{e}_{2} .
\end{aligned}
$$

Notice that along $S$

$$
\begin{aligned}
\vec{n} \times\left(\vec{H}_{0}^{1}-\vec{H}_{0}^{2}\right) e^{-i \alpha x_{1}} & =a\left(x_{1}\right) \vec{\tau}\left(x_{1}\right), \\
\vec{n} \times\left(\vec{E}_{0}^{1}-\vec{E}_{0}^{2}\right) e^{-i \alpha x_{1}} & =b\left(x_{1}\right) \vec{e}_{2} .
\end{aligned}
$$

If we take the scalar product of the equations (7.23) with $\vec{e}_{2}$ and $\vec{\tau}\left(x_{1}\right)$ respectively, we get a system of linear homogeneous equations for $I_{2}, J_{\tau}$ of the form

$$
I_{2}=W_{1}\left(I_{2}, J_{\tau}\right), \quad J_{\tau}=W_{2}\left(I_{2}, J_{\tau}\right) .
$$

Similarly, if we multiply the equations in (7.23) scalarly by $\vec{\tau}\left(x_{1}\right)$ and $\vec{e}_{2}$, respectively, we get a system of linear integral equations of the form

$$
\begin{aligned}
& I_{\tau}=a+W_{3}\left(I_{\tau}, J_{2}\right), \\
& J_{2}=b+W_{4}\left(I_{\tau}, J_{2}\right) .
\end{aligned}
$$


If the first Fredholm alternative holds for (7.23) then the first Fredholm alternative holds for both (8.3), (8.4). Therefore we must have

$$
I_{2}=0, \quad J_{\tau}=0 \text {. }
$$

Of course, the trivial solution (8.5) is always a solution of (8.3). Thus, in seeking a solution of (7.23), it seems reasonable to always choose $I_{2}=0, J_{\tau}=0$ and concentrate just on finding a solution to (8.4).

Theorem 8.1. If the first Fredholm alternative holds for (7.23), then the unique solution has the form

$$
\vec{I}=I_{\tau} \vec{\tau}, \quad \vec{J}=J_{2} \vec{e}_{2}
$$

and the solution of (1.1)-(1.4) has the form

$$
\vec{E}=E_{1} \vec{e}_{1}+E_{3} \vec{e}_{3}, \quad \vec{H}=H_{2} \vec{e}_{2} \text { outside } S .
$$

Proof. We have already proved (8.6). To prove (8.7), notice that $J_{1}=0$ and $\rho(\vec{J})=0$. It is now easy to check that all the terms on the right-hand side of (4.13) are vectors parallel to $\vec{e}_{2}$. It follows that $\vec{H}=H_{2} \vec{e}_{2}$. Similarly one can check that all the terms on the right-hand side of (4.12) are orthogonal to $\vec{e}_{2}$, and the first assertion of (8.7) thus follows.

\section{UNIQUENESS}

Set

$$
\begin{array}{ll}
\beta_{n}^{1}=\left\{k_{1}^{2}-\left(\alpha-\frac{2 \pi n}{L}\right)^{2}\right\}^{1 / 2}, & \operatorname{Im} \beta_{n}^{1} \geq 0(n=0, \pm 1, \pm 2, \ldots), \\
\beta_{n}^{2}=\left\{k_{2}^{2}-\left(\alpha-\frac{2 \pi n}{L}\right)^{2}\right\}^{1 / 2}, & \operatorname{Im} \beta_{n}^{2} \geq 0(n=0, \pm 1, \pm 2, \ldots) .
\end{array}
$$

From (7.1), the representation (4.12), (4.13) and Theorem 3.2 we deduce that (9.2)

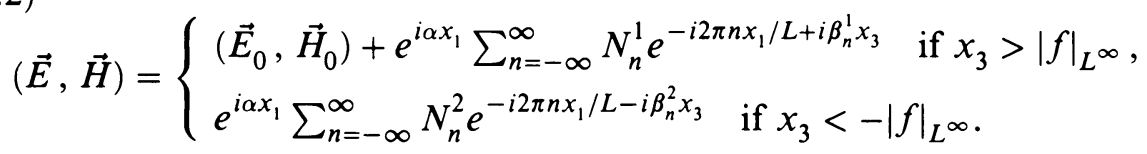

and $\left|N_{n}^{j}\right| \leq C / \sqrt{1+|n|}$. The coefficients $N_{n}^{1}$ are called the reflection coeffcients, and the coefficients $N_{n}^{2}$ are called the transmission coefficients.

Theorem 9.1. Suppose $(\vec{E}, \vec{H})$ is a solution of the Maxwell equations (1.1), (1.2) outside $S$, satisfying (1.3), (1.4) such that $e^{-i \alpha x_{1}}(\vec{E}, \vec{H})$ is independent of $x_{2}$ and is periodic in $x_{1}$ of period $L$. If $(\vec{E}, \vec{H})$ has the form (9.2) in $\left\{\left|x_{3}\right|>|f|_{L^{\infty}}\right\}$ with vectors $N_{n}^{j}$ uniformly bounded, then $(\vec{E}, \vec{H})$ is unique provided the first Fredholm alternative holds the system (7.23). 
Proof. Suppose there are two solutions and denote by their difference $(E, H)$. Then

$$
e^{-i \alpha x_{1}}(E, H)= \begin{cases}\sum_{n=-\infty}^{\infty} M_{n}^{1} e^{-i 2 \pi n x_{1} / L+i \beta_{n}^{1} x_{3}} & \text { if } x_{3}>|f|_{L^{\infty}}, \\ \sum_{n=-\infty}^{\infty} M_{n}^{2} e^{-i 2 \pi n x_{1} / L-i \beta_{n}^{2} x_{3}} & \text { if } x_{3}<-|f|_{L^{\infty}} .\end{cases}
$$

Set $\vec{I}=-\vec{n} \times \vec{H}, \vec{J}=\vec{n} \times \vec{E}, \vec{I}_{\alpha}=\vec{I} e^{-i \alpha x_{1}}, \vec{J}_{\alpha}=\vec{J} e^{-i \alpha x_{1}}$ and define

$$
\begin{aligned}
F(\vec{I}(y), & \left.\vec{J}(y), \Psi_{k}(x-y)\right) \\
=\{ & \frac{i \omega \mu}{c} \vec{I}(y) \Psi_{k}(x-y)-\vec{J}(y) \times \nabla_{y} \Psi_{k}(x-y) \\
& \quad+\frac{c}{i \omega \varepsilon} \nabla_{x}\left[I(y) \cdot \nabla_{y} \Psi_{k}(x-y)\right]+i \alpha \vec{J}(y) \times \vec{e}_{1} \Psi_{k}(x-y) \\
& +\frac{c \alpha}{\omega \varepsilon}\left[\vec{I}(y) \cdot \nabla_{y} \Psi_{k}(x-y) \vec{e}_{1}+I_{1} \nabla_{y} \Psi_{k}(x-y)\right] \\
& \left.\quad-\frac{i c \alpha^{2}}{\omega \varepsilon} \vec{e}_{1} I_{1}(y) \Psi_{k}(x-y)\right\}
\end{aligned}
$$

If we use the integral representation [7, p. 130] for $\vec{E}, \vec{H}$ in

$$
D_{m}=\left\{f\left(x_{1}\right)<x_{3}<Y,-m L<x_{1}<m L\right\},
$$

where $Y>|f|_{L^{\infty}}$, then we obtain a representation similar to (2.3), (2.4) with $S$ replaced by $\partial D_{m}$. Integrating with respect to $y_{2},-\infty<y_{2}<\infty$, and then letting $m \rightarrow \infty$ we find (since

$$
H_{0}^{(1)}(z) \sim \sqrt{\frac{2}{\pi z}} e^{i(z-\pi / 4)} \text { as } z \rightarrow \infty ;
$$

e.g. $[6$, p. $962, \S 8451, \# 3])$ that the boundary integrals over $x_{1}= \pm m L$ converge to zero. To the remaining integrals (on $S$ and on $y_{3}=Y$ ) we apply the process which led from (2.3), (2.4) to (4.7), (4.8). We thus obtain the representation

$$
e^{-i \alpha x_{1}} \vec{E}(x)=\int_{\partial D_{Y}} F\left(\vec{I}(y), \vec{J}(y), \Psi_{1}(x-y)\right) d s
$$

where $D_{Y}=\left\{\left(x_{1}, x_{3}\right) ; 0<x_{1}<L, f\left(x_{1}\right)<x_{3}<Y\right\}$. By periodicity, the integrals over $x_{1}=0$ and $x_{1}=L$ cancel each other. Therefore

$$
\begin{aligned}
e^{-i \alpha x_{1}} & \vec{E}(x)-\int_{0}^{L} F\left(\vec{I}, \vec{J}, \Psi_{1}(x-y)\right)\left(y_{1}, f\left(y_{1}\right)\right) \sigma\left(y_{1}\right) d y_{1} \\
= & -\int_{0}^{L} F\left(\vec{I}, \vec{J}, \Psi_{1}(x-y)\right)\left(y_{1}, Y\right) d y_{1} .
\end{aligned}
$$

By Theorem 3.2 and (9.3), the left-hand side has the form

$$
\sum K_{n} e^{i \alpha_{n} x_{1}+i \beta_{n}^{1} x_{3}} \quad\left(\alpha_{n}=\frac{2 \pi n}{L}\right),
$$


whereas, by Theorem 3.2, the right-hand side of (9.4) has the form

$$
\sum R_{n} e^{i \alpha_{n} x_{1}-i \beta_{n}^{1} x_{3}}
$$

where $R_{n}=R_{n}(Y)$. Letting $Y \rightarrow \infty$, we get

$$
\sum K_{n} e^{i \alpha_{n} x_{1}+i \beta_{n}^{1} x_{3}}=\sum R_{n}(\infty) e^{i \alpha_{n} x_{1}-i \beta_{n}^{1} x_{3}}
$$

for all $x_{3}>|f|_{L^{\infty}}$. It follows that $K_{n} e^{i \beta_{n}^{1} x_{3}}=R_{n}(\infty) e^{-i \beta_{n}^{1} x_{3}}$ for all $x_{3}>$ $|f|_{L^{\infty}}$, which implies that $K_{n}=R_{n}(\infty)=0$. Thus, the right-hand side of (9.4) converges to zero as $Y \rightarrow \infty$, and we obtain

$$
e^{-i \alpha x_{1}} \vec{E}(X)=\int_{0}^{L} F\left(\vec{I}, \vec{J}, \Psi_{1}(x-y)\right)\left(y_{1}, f\left(y_{1}\right)\right) \sigma\left(y_{1}\right) d y_{1} .
$$

This representation is the same as (4.7), and similarly we derive a representation for $\vec{H}$ as in (4.8). A similar representation holds in $\left\{x_{3}<-f\right\}$. Letting $x_{3} \rightarrow f\left(x_{1}\right)$ and using the jump relations derived in $\S 5$, we find that $\vec{I}, \vec{J}$ must satisfy the homogeneous version of the system of integral equations (7.23). Since for this system the first Fredholm alternative holds, $\vec{I}=\vec{J}=0$ and therefore $\vec{E}=\vec{H}=0$.

\section{Piecewise SMooth interface}

In this section we extend the results of the previous sections to the case where $S$ is piecewise smooth and is not necessarily a graph in the $x_{3}$-direction. We take $S$ to have the form

$(10.1)$ $S=\left\{\left(x_{1}, x_{2}, x_{3}\right) ; x_{1}=f_{1}(s), x_{3}=f_{3}(s),-\infty<s<\infty\right.$ and $\left.-\infty<x_{2}<\infty\right\}$, where $s$ is the length parameter, and assume that for some $l_{0}>0, L>0$,

$$
\begin{aligned}
& f_{1}\left(s+n l_{0}\right)=n L+f_{1}(s), \\
& f_{3}\left(s+n l_{0}\right)=f_{3}(s) \quad(-\infty<s<\infty, n= \pm 1, \pm 2, \ldots),
\end{aligned}
$$

Denoting by $s_{1}<s_{2}<\cdots<s_{n}$ the points of discontinuity of the derivatives of $f_{j}$ in the interval $0 \leq s \leq l_{0}$ and setting $s_{n+1}=l_{0}+s_{1}$, we further assume that

$$
f_{j}(s) \text { is in } C^{2}\left[s_{i}, s_{i+1}\right] \text { for } 1 \leq i \leq n .
$$

Notice that our assumption on $S$ include the case where $S \cap\left\{x_{2}=0\right\}$ is a step-function, i.e., $x_{3}=f\left(x_{1}\right)$ where $f$ is piecewise constant.

Set $l=S \cap\left\{x_{2}=0\right\}, \vec{l}(s)=\left(f_{1}(s), 0, f_{3}(s)\right)$ and introduce the tangent $\vec{\tau}=d \vec{l} / d s$ and normal $\vec{n}=\vec{e}_{2} \times \vec{\tau}$. We shall also write $y(t)=\vec{l}(t)$. 
We begin with the representation (2.3), (2.4), but take $\vec{I}, \vec{J}$ to have the form

$$
\begin{aligned}
\vec{I}(y(t)) & \equiv \vec{I}(t)=I(t) \vec{\tau}(t), & I(t) & \equiv I(y(t)), \\
\vec{J}(y(t)) & \equiv \vec{J}(t)=J(t) \vec{e}_{2}, & J(t) & \equiv J(y(t)) ;
\end{aligned}
$$

the general case where

$$
\begin{aligned}
& \vec{I}=I(t) \vec{\tau}(t)+I_{2}(t) \vec{e}_{2}, \\
& \vec{J}=J(t) \vec{e}_{2}+J_{\tau}(t) \vec{\tau}(t)
\end{aligned}
$$

can be handled similarly. However, as in $\S 8$, we can derive a system of equation for $(I, J)$ and (separately) for $\left(I_{2}, J_{\tau}\right)$, and the system for $I_{2}, J_{\tau}$ has a solution $I_{2}=0, J_{\tau}=0$.

Assuming (10.5) we have

$$
\begin{gathered}
J(t) \nabla_{y} \Psi_{k}(x-y(t))=J(t) \vec{e}_{2} \cdot\left(\partial_{y_{1}} \Psi_{k} \vec{e}_{1}+\partial_{y 3} \Psi_{k} \vec{e}_{3}\right)=0, \\
\rho(\vec{I}(t))=\frac{d}{d t}\left[\sigma(y(t)) \vec{I}(y(t)) \cdot \vec{e}_{1}\right]=\frac{d I(t)}{d t} .
\end{gathered}
$$

Proceeding with (2.4) and using (10.5), (10.7) we obtain analogously to (4.8):

$$
\begin{aligned}
H_{\alpha}(x)=\int_{0}^{l_{0}}\left\{\frac{i \omega \varepsilon}{c} \vec{J} \Psi_{k}(x-y(t))+\vec{I}(t) \times \nabla_{y} \Psi_{k}(x-y(t))\right. \\
\\
\left.\quad-i \alpha\left(\vec{I}(t) \times \vec{e}_{1}\right) \Psi_{k}(x-y(t))\right\} d t .
\end{aligned}
$$

Similarly, proceeding with (2.4) and using (10.5), (10.8) we obtain analogously to $(4.12)$ :

$(10.10)$

$$
\begin{aligned}
E_{\alpha}(x)= & \int_{0}^{l_{0}}\left\{\frac{i \omega \mu}{c} \vec{I}(t) \Psi_{k}(x-y(t))-\vec{J}(t) \times \nabla_{y} \Psi_{k}(x-y(t))\right. \\
& \left.+\frac{c}{i \omega \varepsilon} \frac{d I(t)}{d t} \nabla_{y} \Psi_{k}(x-y(t))\right\} d t \\
& +\int_{0}^{l_{0}}\left\{i \alpha \vec{J}(t) \times \vec{e}_{1}-\frac{c \alpha}{\omega \varepsilon} \frac{d I(t)}{d t} \vec{e}_{1}-\frac{i c \alpha^{2}}{\omega \varepsilon} I(t) \vec{e}_{1}(t)\right\} \Psi_{k}(x-y(t)) d t \\
& +\frac{c \alpha}{\omega \varepsilon} \int_{0}^{l_{0}} I(t) \nabla_{y} \Psi_{k}(x-y(t)) d t .
\end{aligned}
$$

We set

$$
\Sigma=\left\{0, s_{1}, \ldots, s_{n}\right\} .
$$

Lemma 10.1. If $s \notin \Sigma$ then, for any $u \in L^{2}\left(0, l_{0}\right)$,

Proof. We have

$$
\lim _{\varepsilon \rightarrow 0} \int_{\{|t-s|>\varepsilon\}}^{l_{0}} u(t) \frac{\vec{l}(s)-\vec{l}(t)}{|\vec{l}(s)-\vec{l}(t)|^{2}} d t \text { exists. }
$$

$$
\vec{l}(s)-\vec{l}(t)=\left(f_{1}^{\prime}(s) \vec{e}_{1}+f_{3}^{\prime}(s) \vec{e}_{3}\right)(s-t)+(s-t)^{2} F(t),
$$


where $F(t)$ is continuous in $t$. Hence

$$
\frac{\vec{l}(s)-\vec{l}(t)}{|\vec{l}(s)-\vec{l}(t)|^{2}}=\frac{f_{1}^{\prime}(s) \vec{e}_{1}+f_{3}^{\prime}(s) \vec{e}_{3}}{s-t}+F_{1}(t),
$$

where $F_{1}(t)$ is continuous in $t$. Using the Hilbert transform properties, the assertion of the lemma follows.

Lemma 10.2. Let $x=\vec{l}(s), s \notin \Sigma$. Then for any $L^{2}$ function $u(t)$,

$$
\begin{aligned}
\lim _{\substack{z \rightarrow \pm 0 \\
z \neq 0}} \int_{0}^{l_{0}} u(t) \nabla_{y} \Psi_{k}(x+z \vec{n}(s)-y(t)) d t \\
= \pm \frac{1}{2} \vec{n}(s) u(s)+\frac{1}{2 \pi} \text { p.v. } \int_{0}^{l_{0}} u(t) \frac{\vec{l}(s)-\vec{l}(t)}{|\vec{l}(s)-\vec{l}(t)|^{2}} d t \\
\quad+\int_{0}^{l_{0}} u(t) \nabla_{y}\left(\Psi_{k}(x-y(t))-\frac{1}{2 \pi} \log \frac{1}{|x-y(t)|}\right) d t
\end{aligned}
$$

holds for a.a. s.

Proof. Since

$$
\widetilde{\Psi}_{k}(x-y) \equiv \Psi_{k}(x-y)-\frac{1}{2 \pi} \log \frac{1}{|x-y|}
$$

is a continuous function, it suffices to prove that

$$
\begin{aligned}
& \lim _{\substack{z \rightarrow \pm 0 \\
z \neq 0}} \frac{1}{2 \pi} \int_{0}^{l_{0}} u(t) \frac{\vec{l}(s)-\vec{l}(t)+z \vec{n}(s)}{|\vec{l}(s)-\vec{l}(t)+z \vec{n}(s)|^{2}} d t \\
& \quad= \pm \frac{1}{2} \vec{n}(s) u(s)+\frac{1}{2 \pi} \text { p.v. } \int_{0}^{l_{0}} u(t) \frac{\vec{l}(s)-\vec{l}(t)}{|\vec{l}(s)-\vec{l}(t)|^{2}} d t
\end{aligned}
$$

Since

$$
\begin{aligned}
|\vec{l}(s)-\vec{l}(t)+z \vec{n}(s)|^{2} & =|\vec{l}(s)-\vec{l}(t)|^{2}+z^{2}+2 z \vec{n}(s) \cdot(\vec{l}(s)-\vec{l}(t)) \\
& =(s-t)^{2}+z^{2}+z O\left((s-t)^{2}\right)+O\left((s-t)^{3}\right) \quad(\text { by (10.12)) }
\end{aligned}
$$

we have

$$
\frac{\vec{l}(s)-\vec{l}(t)}{|l(s)-l(t)+z \vec{n}(s)|^{2}}=\frac{(s-t) \vec{\tau}(s)}{(s-t)^{2}+z^{2}}+\Delta(s, t, z)
$$


where $\Delta(s, t, z)$ is continuous in all variables. Hence

$(10.14)$

$$
\begin{aligned}
\lim _{z \rightarrow \pm 0} & \int_{0}^{l_{0}} u(t) \frac{\vec{l}(s)-\vec{l}(t)}{|\vec{l}(s)-\vec{l}(t)+z \vec{n}(s)|^{2}} d t \\
= & \lim _{z \rightarrow \pm 0} \int_{0}^{l_{0}} \frac{(s-t) \vec{\tau}(s) u(s)}{(s-t)^{2}+z^{2}} d t+\lim _{z \rightarrow \pm 0} \int_{0}^{l_{0}} u(t) \Delta(s, t, z) d t \\
& +\lim _{z \rightarrow \pm 0} \int_{0}^{l_{0}} \frac{(s-t) \vec{\tau}(s)(u(t)-u(s))}{(s-t)^{2}+z^{2}} d t \\
= & \text { p.v. }\left[\int_{0}^{l_{0}} \frac{(s-t)(u(t)-u(s))}{(s-t)^{2}} d t\right] \vec{\tau}(s)+\int_{0}^{l_{0}} u(t) \Delta(s, t, 0) d t \\
= & \text { p.v. } \int_{0}^{l_{0}} u(t)\left[\frac{1}{s-t} \vec{\tau}(s)+\Delta(s, t, 0)\right] d t \\
= & \text { p.v. } \int_{0}^{l_{0}} \frac{\vec{l}(s)-\vec{l}(t)}{|\vec{l}(s)-l(t)|^{2}} u(t) d t,
\end{aligned}
$$

by reversing the previous steps in the case $z=0$. Next

$$
\frac{z \vec{n}(s)}{|\vec{l}(s)-\vec{l}(t)+z \vec{n}(s)|^{2}}=z \vec{n}(s)\left[\frac{1}{(s-t)^{2}+z^{2}}+O\left(\frac{z+|s-t|}{(s-t)^{2}+z^{2}}\right)\right]
$$

and therefore

$$
\begin{aligned}
\lim _{z \rightarrow \pm 0} \int_{0}^{l_{0}} \frac{z \vec{n}(s)}{|\vec{l}(s)-\vec{l}(t)+z u(s)|^{2}} d t & =\vec{n}(s) \lim _{z \rightarrow \pm 0} \int_{0}^{l_{0}} \frac{u(t) z}{(s-t)^{2}+z^{2}} d t \\
& = \pm \pi \vec{n}(s) u(s) .
\end{aligned}
$$

Combining this with (10.14), the assertion (10.13) follows.

Using Lemma 10.2 we can now deduce from (10.9), (10.10) that

$$
\vec{E}^{ \pm}(x)=\lim _{z \rightarrow \pm 0} \vec{E}_{\alpha}(x+z \vec{n}(s)), \quad \vec{H}^{ \pm}(x)=\lim _{z \rightarrow \pm 0} \vec{H}_{\alpha}(x+z \vec{n}(s))
$$

exist for any $x=x(s), s \notin \Sigma$, and, setting $\vec{E}^{ \pm}(s)=\vec{E}^{ \pm}(x(s)), \vec{H}^{ \pm}(s)=$ $\vec{H}^{ \pm}(x(s))$, we easily get

$(10.15)$

$$
\begin{aligned}
\vec{n}(s) \times\left(H^{+}(s)-H^{-}(s)\right) & \\
=\{I(s) & +\frac{i \omega}{c} \int_{0}^{l_{0}}\left(\varepsilon_{1} \Psi_{1}-\varepsilon_{2} \Psi_{2}\right)(x(s)-y(t)) J(t) d t \\
\quad+\int_{0}^{l_{0}} I(t)\left(f_{3}^{\prime}(t) \frac{\partial}{\partial y_{1}}-f_{1}^{\prime}(t) \frac{\partial}{\partial y_{3}}\right)\left(\Psi_{1}-\Psi_{2}\right)(x(s)-y(t)) d t & \left.\quad-\int_{0}^{l_{0}} i \alpha I(t)\left(\Psi_{2}-\Psi_{1}\right)(x(s)-y(s)) f_{3}^{\prime}(t) d t\right\} \vec{\tau}(s),
\end{aligned}
$$


and (using also (10.13))

$(10.16)$

$$
\begin{aligned}
& \vec{n}(s) \times\left(\vec{E}^{+}(s)-\vec{E}^{-}(s)\right) \\
& =\left\{-J(s)+\frac{c}{2 \pi \omega i}\left(\frac{1}{\varepsilon_{2}}-\frac{1}{\varepsilon_{1}}\right) \int_{0}^{l_{0}}\left[\frac{d I(t)}{d t}+i \alpha I(t)\right]\right. \\
& \cdot \frac{f_{1}^{\prime}(s)\left(f_{1}(s)-f_{1}(t)\right)+f_{3}^{\prime}(s)\left(f_{3}(s)-f_{3}(t)\right)}{|\vec{l}(s)-\vec{l}(t)|^{2}} d t \\
& +\frac{c}{i \omega} \int_{0}^{l_{0}}\left[\frac{d I(t)}{d t}+i \alpha I(t)\right]\left(f_{1}^{\prime}(s) \frac{\partial}{\partial y_{1}}+f_{3}^{\prime}(s) \frac{\partial}{\partial y_{3}}\right) \\
& \cdot\left(\frac{\widetilde{\Psi}_{2}}{\varepsilon_{2}}-\frac{\widetilde{\Psi}_{1}}{\varepsilon_{1}}\right)(x(s)-y(t)) d t \\
& +\int_{0}^{l_{0}} J(t)\left[f_{1}^{\prime}(s) \frac{\partial}{\partial y_{3}}-f_{3}^{\prime}(s) \frac{\partial}{\partial y_{1}}\right]\left(\Psi_{1}-\Psi_{2}\right)(x(s)-y(t)) d t \\
& +\frac{c \alpha}{\omega} \int_{0}^{l_{0}}\left[\frac{d I(t)}{d t}+i \alpha I(t)\right] f_{1}^{\prime}(s)\left(\frac{\Psi_{2}}{\varepsilon_{2}}-\frac{\Psi_{1}}{\varepsilon_{1}}\right)(x(s)-y(t)) d t \\
& +\int_{0}^{l_{0}}\left[i \alpha f_{3}^{\prime}(s) J(t)-\frac{i \omega \mu}{c}\left[f_{1}^{\prime}(s) f_{1}^{\prime}(t)+f_{3}^{\prime}(s) f_{3}^{\prime}(t)\right] I(t)\right] \\
& \left.\cdot\left(\Psi_{1}-\Psi_{2}\right)(x(s)-y(t)) d t\right\} \vec{e}_{2} .
\end{aligned}
$$

Following the procedure of $\S 7$ we now wish to substitute $I$ from $(10.15)$ into (10.16) in order to get rid of the derivatives $d I / d t$ in (10.16). We require here a lemma analogous to Lemma 7.2 whose proof uses Lemma 10.2:

Lemma 10.3. If

$$
L u=\int_{0}^{l_{0}} u(t)\left(\varepsilon_{1} \Psi_{1}-\varepsilon_{2} \Psi_{2}\right)(x(s)-y(t)) d t
$$

then

$$
\begin{aligned}
\left(\frac{d}{d s} L u\right)(s)= & \varepsilon_{2} \frac{d}{d s} \int_{0}^{l_{0}} u(t)\left(\Psi_{1}-\Psi_{2}\right)(x(s)-y(t)) d t \\
& +\frac{\varepsilon_{2}-\varepsilon_{1}}{2 \pi} \text { p.v. } \int_{0}^{l_{0}} u(t) \frac{\vec{\tau}(s) \cdot(\vec{l}(s)-\vec{l}(t))}{|\vec{l}(s)-\vec{l}(t)|^{2}} d t \\
& +\left(\varepsilon_{2}-\varepsilon_{1}\right) \int_{0}^{l_{0}} u(t)\left(f_{1}^{\prime}(s) \frac{\partial}{\partial y_{1}}+f_{3}^{\prime}(s) \frac{\partial}{\partial y_{3}}\right) \widetilde{\Psi}_{1}(x(s)-y(t)) d t .
\end{aligned}
$$

The proof is omitted.

Introduce the operator $\widetilde{H}$ :

$$
\widetilde{H} u(s)=\frac{1}{\pi} \text { p.v. } \int_{0}^{l_{0}} u(t) \frac{\vec{\tau}(s) \cdot(\vec{l}(s)-\vec{l}(t))}{|\vec{l}(s)-\vec{l}(t)|^{2}} d t .
$$


If we substitute $I$ from (10.15) into (10.16) and use Lemma 10.3 and the notation (10.17), we get a system

$$
\begin{gathered}
I+\widetilde{T}_{1}(I, J)=-I_{0}, \\
J-\frac{\left(\varepsilon_{1}-\varepsilon_{2}\right)^{2}}{4 \varepsilon_{1} \varepsilon_{2}} \widetilde{H}^{2}(J)+\widetilde{T}_{2}(I, J)=-\widetilde{J}_{0}
\end{gathered}
$$

where $I_{0} \vec{\tau}(s)=-\vec{n}(s) \times\left(\vec{H}_{0}^{+}(s)-\vec{H}_{0}^{-}(s)\right)$ and $\widetilde{J}_{0}$ depends on both $I_{0}$ and $\vec{n} \times\left(\vec{E}_{0}^{+}-\vec{E}_{0}^{-}\right) e^{-i \alpha x_{1}}$; for any $1<p<\infty$ the operators $\widetilde{T}_{1}, \widetilde{T}_{2}$ are compact integral operators in the space $X_{p}$ of $L^{p} \quad l_{0}$-periodic functions defined on the curve $S \cap\left\{x_{2}=0\right\}$ with the $L^{p}\left(0, l_{0}\right)$ norm.

Thus the only essential difference between (10.18) and (7.21), (7.22) or (8.4) is that in the previous system $\widetilde{H}$ was the bounded operator $H$ (the Hilbert transform) satisfying $H^{2}=-1$ whereas now we have an operator $\widetilde{H}$ which is not as "nice" as the Hilbert transform. We nonetheless have

Lemma 10.4. $\widetilde{H}$ is a bounded operator in $X_{p}$, that is,

$$
\|\widetilde{H} u\|_{L^{p}\left(0, l_{0}\right)} \leq C\|u\|_{L^{p}\left(0, l_{0}\right)} .
$$

Proof. We first consider the behavior of $(\widetilde{H} u)(s)$ for $s$ near a point $s_{i}$ where $\vec{\tau}(s)$ has a discontinuity. For simplicity we take $0<s_{i}<l_{0}$. (If $s_{i}=0$ we can work with $\int_{-l_{0}}^{0}$ instead of $\int_{0}^{l_{0}}($ in $(10.17))$, since $u$ and $l$ are $l_{0}$-periodic. Set

$$
\vec{\alpha}=\vec{\tau}\left(s_{i}+0\right)-\vec{\tau}\left(s_{i}-0\right) .
$$

Suppose $s_{i}<s<s_{i+1}$ and consider the portion of the integral $(\widetilde{H} u)(s)$ from $t=s_{i}$ to $t=s_{i+1}$. Then the integrand satisfies

$$
u(t) \frac{\vec{\tau}(s) \cdot(\vec{l}(s)-\vec{l}(t))}{|\vec{l}(s)-\vec{l}(t)|^{2}}=\frac{u(t)}{s-t}+u(t) F_{0}, \quad F_{0} \text { bounded }
$$

(since $\tau(\cdot)$ is in $\left.C^{1}\left[s_{i}, s_{i+1}\right]\right)$.

Next consider the portion of the integral $(\widetilde{H} u)(s)$ for $s_{i-1}<t<s_{i}$. Introduce the auxiliary $C^{1,1}$ curve $\vec{l}_{*}(t)$ in $\left[s_{i-1}, s_{i+1}\right]$ defined by

$$
\begin{aligned}
& \vec{l}_{*}=\vec{l}(t) \quad \text { if } s_{i}<t<s_{i+1}, \\
& \vec{l}_{*}=\vec{l}(t)+\vec{\alpha}\left(t-s_{i}\right) \quad \text { if } s_{i-1}<t<s_{i} .
\end{aligned}
$$

Then

$$
\frac{\vec{\tau}(s) \cdot(\vec{l}(s)-\vec{l}(t))}{|\vec{l}(s)-\vec{l}(t)|^{2}}=\frac{\vec{\tau}(s) \cdot\left[\vec{\tau}(s)(s-t)-\vec{\alpha}\left(t-s_{i}\right)+O(s-t)^{2}\right]}{\left|\vec{\tau}(s)(s-t)-\vec{\alpha}\left(t-s_{i}\right)+O(s-t)^{2}\right|^{2}} .
$$

Notice that for $s>s_{i}, \vec{\tau}(s)=\vec{\tau}\left(s_{i}+0\right)+O\left(s-s_{i}\right)$. Since also $\vec{\tau}\left(s_{i}+0\right)$. $\vec{\tau}\left(s_{i}-0\right)>-1$, the denominator in the last fraction is $\geq c\left[\left(s-s_{i}\right)^{2}+\left(t-s_{i}\right)^{2}\right]$ 
$(c>0)$. It now easily follows that, for $s_{i-1}<t<s_{i}$,

$$
\begin{aligned}
& \left|u(t) \frac{\vec{\tau}(s) \cdot(\vec{l}(s)-\vec{l}(t))}{|\vec{l}(s)-\vec{l}(t)|}-\frac{u(t)}{s-t}\right| \leq C \frac{\left|t-s_{i}\right|+(s-t)^{2}}{\left(s-s_{i}\right)^{2}+\left(t-s_{i}\right)^{2}}|u(t)| \\
& \quad \leq C\left(1+\frac{s_{i}-t}{\left(s-s_{i}\right)^{2}+\left(t-s_{i}\right)^{2}}\right)|u(t)| \\
& \quad \leq C\left(1+\frac{2}{s-t}\right)|u(t)|
\end{aligned}
$$

since $t<s_{i}<s$.

We have thus proved that, for $s_{i}<s<s_{i+1}$,

$$
\begin{aligned}
& \left|\int_{s_{i-1}}^{s_{i+1}} u(t) \frac{\vec{\tau}(s) \cdot(\vec{l}(s)-\vec{l}(t))}{|\vec{l}(s)-\vec{l}(t)|^{2}} d t\right| \\
& \quad \leq C\left|\int_{s_{i-1}}^{s_{i}} \frac{|u(t)|}{t-s} d t\right|+C \int_{s_{i-1}}^{s_{i+1}}|u(t)| d t+\left|\int_{s_{i-1}}^{s_{i+1}} \frac{u(t)}{s-t} d t\right| \\
& \quad=C H\left(|u| \chi_{\left[s_{i-1}, s_{i}\right]}\right)(s)+C \int_{s_{i-1}}^{s_{i+1}}|u(t)| d t+\left|H\left(u \chi_{\left[s_{i-1}, s_{i+1}\right.}\right)\right| .
\end{aligned}
$$

A similar estimate holds for $s$ in the interval $\left(s_{i-1}, s_{i}\right)$ and clearly also for $s$ in $\left(0, s_{i-1}\right)$ or in $\left(s_{i+1}, l_{0}\right)$. Since $H$ is a bounded operator in $L^{p}$, the assertion of the lemma follows.

We summarize:

Theorem 10.5. Let $S$ be given by (10.1)-(10.4). Then there exists a solution of (1.1)-(1.4) of the form (8.7) if there exists a solution $I, J$ of (10.18), where $\widetilde{T}_{1}, \widetilde{T}_{2}$ are compact linear integral operators in $X_{p}$ and $\widetilde{H}$ is a bounded linear operator in $X_{p}$, given by (10.17); here $p$ is any number satisfying $1<p<\infty$.

Remark 10.1. Theorem 9.1 extends to the case where $S$ satisfies (10.1)-(10.4); thus, the solution having the form (9.2) is unique if the system (10.18) has a unique solution.

In order to show that the system (10.18) is a Fredholm system of equations we need to analyze $\widetilde{H}^{2}$ more carefully. We shall be working with the spaces $X_{p}, 1 \leq p \leq 2$; if $T$ is bounded linear operator from $X_{p}$ to $X_{p}$ then its norm is denoted by $\|T\|_{L^{p}}$.

Lemma 10.6. For any $u \in X_{1} \cap X_{2}$

$$
\widetilde{H}^{2} u=-u+D u+T u,
$$

where $D$ is a compact operator from $L^{p}\left(0, l_{0}\right)$ into $L^{p}\left(0, l_{0}\right)$ for $1 \leq p \leq 2$, and

$$
\|T u\|_{L^{1}\left(0, l_{0}\right)} \leq \frac{1}{4}\|u\|_{L^{1}\left(0, l_{0}\right)},
$$




$$
\|T u\|_{L^{2}\left(0, l_{0}\right)} \leq \frac{3+2 \sqrt{2}}{4}\|u\|_{L^{2}\left(0, l_{0}\right)} .
$$

Proof. Using a partition of unity to write

$$
u=\sum \chi_{i} u
$$

where $\chi_{i}$ is supported $\left\{s_{i-1}+2 \delta, s_{i+1}-2 \delta\right\}$ for some $\delta>0$, it is sufficient to concentrate on $\tilde{H}^{2}\left(\chi_{i} u\right)$. Setting

$$
L_{0}^{p}=\left\{u \in L^{p}\left(s_{i-1}+\delta, s_{i+1}-\delta\right) ; u(t)=0 \text { if } t<s_{i-1}+2 \delta \text { or if } t>s_{i+1}-2 \delta\right\}
$$

it is sufficient to establish $(10.21)-(10.23)$ for $u$ in $L_{0}^{1} \cap L_{0}^{2}$. (Here we have taken for simplicity $0 \leq s_{i-1}, s_{i+1} \leq l_{0}$; if $s_{i}$ is the smallest or largest point in $\Sigma$, then some small modifications need to be made, using the $l_{0}$-periodicity of the functions $u(t)$.)

To simplify the notation we take

(10.24) $s_{i-1}=-1, \quad s_{i-1}+2 \delta=-\frac{1}{2}, \quad s_{i}=0, \quad s_{i+1}-2 \delta=\frac{1}{2}, \quad s_{i+1}=1$.

We first consider a special case where

$$
\vec{l}(s)= \begin{cases}\vec{\alpha} s & \text { if }-1<s<0, \\ \vec{\beta} s & \text { if } 0<s<1,\end{cases}
$$

and $|\vec{\alpha}|=|\vec{\beta}|=1, \vec{\alpha} \neq \vec{\beta}$.

Lemma 10.7. The assertions of Lemma 10.6 holds for the case where $u$ varies in $L_{0}^{p}$ and $\widetilde{H}^{2} u$ is considered in $L^{p}\left(0, l_{0}\right)$, provided $(10.24),(10.25)$ hold. Proof. We shall use complex notation

$$
\vec{\alpha}=e^{i \theta} \equiv \alpha, \quad \vec{\beta}=e^{i \varphi} \equiv \beta
$$

and set

$$
\begin{gathered}
A=\vec{l}(-1), \quad B=\vec{l}(0), \quad C=\vec{l}(1), \\
\zeta(s)= \begin{cases}\alpha s & \text { if }-1<s<0, \\
\beta s & \text { if } 0<s<1 .\end{cases}
\end{gathered}
$$

We assume for definiteness that $\overline{A C}$ lies in $\Omega_{1}$ (i.e., above the curve $S$ ), and denote by $\Gamma$ the boundary of the triangle $A, B, C$.

Since

$$
\vec{\alpha} \cdot \vec{l}=\operatorname{Re} \alpha \vec{\zeta}, \quad \vec{\beta} \cdot \vec{l}=\operatorname{Re} \beta \bar{\zeta},
$$

we can write

$$
\pi \widetilde{H} u(s)= \begin{cases}\operatorname{Re} \int_{-1}^{1} \frac{\alpha u(t)}{\zeta(s)-\zeta(t)} d t, & -1<s<0 \\ \operatorname{Re} \int_{-1}^{1} \frac{\beta u(t)}{\zeta(s)-\zeta(t)} d t, & 0<s<1\end{cases}
$$

Define

$$
\begin{gathered}
F(z)=\int_{-1}^{1} \frac{u(t)}{z-\zeta(t)} d t \quad \text { for } z \in \Omega_{1}, \\
F(\zeta(s))=\lim _{\varepsilon \rightarrow 0+} F(\zeta(s)+\varepsilon n(s)),
\end{gathered}
$$


where

$$
n(s)= \begin{cases}i \alpha & \text { if }-1<s<0, \\ i \beta & \text { if } 0<s<1\end{cases}
$$

is the normal to $S$ pointing into $\Omega_{1}$.

Notice that for $-1<s<0$,

$$
\begin{aligned}
\lim _{\varepsilon \rightarrow 0+} & \operatorname{Re} \int_{-1}^{0} \frac{\alpha u(t)}{\alpha s+i \alpha \varepsilon-\alpha t} d t \\
= & \operatorname{Re} \int_{\{-1,0\} \backslash J} \frac{\alpha u(t)}{\alpha s-\alpha t} d t+\lim _{\varepsilon \rightarrow 0+} \int_{J} \frac{s-t}{(s-t)^{2}+\varepsilon^{2}}[u(t)-u(s)] d t \\
& +\lim _{\varepsilon \rightarrow 0+} \int_{J} \frac{s-t}{(s-t)^{2}+\varepsilon^{2}} u(s) d t
\end{aligned}
$$

where $J$ is a subinterval of $(-1,0)$ symmetric about $s$. The last integral vanishes by symmetry, whereas the limit of the preceding integral is equal a.e. to

We conclude that

$$
\int_{J} \frac{s-t}{(s-t)^{2}}[u(t)-u(s)] d t
$$

$$
\lim _{\substack{z=\zeta(s)+\varepsilon n(s) \\ \varepsilon \rightarrow 0+}} \operatorname{Re} \int_{-1}^{0} \frac{\alpha u(t)}{z-\zeta(t)} d t=\operatorname{Re} \int_{-1}^{0} \frac{\alpha u(t)}{\zeta(s)-\zeta(t)} d t .
$$

Similarly

$$
\lim _{\substack{z=\zeta(s)+\varepsilon n(s) \\ \varepsilon \rightarrow 0+}} \operatorname{Im} \int_{-1}^{0} \frac{\alpha u(t)}{z-\zeta(t)} d t=-\pi u(s) .
$$

Using (10.26) we deduce that, for $-1<s<0$,

$$
\operatorname{Re}(\alpha F)(\zeta(s))=\operatorname{Re} \int_{-1}^{1} \frac{\alpha u(t)}{\zeta(s)-\zeta(t)} d t=\pi \tilde{H} u(s) .
$$

A similar result holds for $0<s<1$; thus

$$
\pi \widetilde{H} u(s)= \begin{cases}\operatorname{Re}(\alpha F)(\zeta(s)) & \text { if }-1<s<0, \\ \operatorname{Re}(\beta F)(\zeta(s)) & \text { if } 0<s<1 .\end{cases}
$$

If $-1<s<0$, then by $(10.28)$

$$
\begin{aligned}
\pi^{2} \widetilde{H}^{2} u(s) & =\pi^{2} \widetilde{H}(\widetilde{H} u)(s) \\
& =\pi \operatorname{Re}\left\{\alpha \int_{-1}^{1} \frac{\widetilde{H} u(t)}{\zeta(s)-\zeta(t)} d t\right\} \\
& =\lim _{\substack{z=\zeta(s)+i \alpha \varepsilon \\
\varepsilon \rightarrow 0+}} \operatorname{Re}\left\{\alpha\left[\int_{-1}^{0} \frac{\operatorname{Re}(\alpha F)(\zeta(t))}{z-\zeta(t)} d t+\int_{0}^{1} \frac{\operatorname{Re}(\beta F)(\zeta(t))}{z-\zeta(t)} d t\right]\right\},
\end{aligned}
$$

where (10.26) was used in the last equality. Since

$$
d \zeta(t)= \begin{cases}a d t & \text { if }-1<t<0 \\ \beta d t & \text { if } 0<t<1\end{cases}
$$


we have

(10.30)

$$
\begin{aligned}
\alpha\left[\int_{-1}^{0}\right. & \left.\frac{\operatorname{Re}(\alpha F)(\zeta(t))}{z-\zeta(t)} d t+\int_{0} \frac{\operatorname{Re}(\beta F)(\zeta(t))}{z-\zeta(t)} d t\right] \\
= & \int_{-1}^{0} \frac{\alpha F(\zeta(t))}{z-\zeta(t)} \alpha d t-\alpha \int_{-1}^{0} \frac{i \operatorname{Im}(\alpha F)(\zeta(t))}{z-\zeta(t)} d t+\int_{0}^{1} \frac{\alpha \operatorname{Re}(\beta F)(\zeta(t))}{z-\zeta(t)} d t \\
= & \left(\oint_{\Gamma} \frac{\alpha F(\zeta)}{z-\zeta} d \zeta-\int_{\overline{B C}+\overline{C A}} \frac{\alpha F(\zeta)}{z-\zeta} d \zeta\right)-\alpha \int_{-1}^{0} \frac{i \operatorname{Im}(\alpha F)(\zeta(t))}{z-\zeta(t)} d t \\
& +\int_{0}^{1} \frac{\alpha \operatorname{Re}(\beta F)(\zeta(t))}{z-\zeta(t)} d t \\
= & \left(-2 \pi i \alpha F(z)-\int_{\overline{C A}} \frac{\alpha F(\zeta)}{z-\zeta} d \zeta-\int_{0}^{1} \frac{\alpha F(\zeta)}{z-\zeta(t)}(\beta d t)\right) \\
& -\alpha \int_{-1}^{0} \frac{i \operatorname{Im}(\alpha F)(\zeta(t))}{z-\zeta(t)} d t+\int_{0}^{1} \frac{\alpha \operatorname{Re}(\beta F)(\zeta(t))}{z-\zeta(t)} d t \\
= & -2 \pi i \alpha(F(z)+G(z))-\int_{0}^{1} \frac{\alpha i \operatorname{Im}(\beta F)(\zeta(t))}{z-\zeta(t)} d t \\
& -\alpha \int_{-1}^{0} \frac{i \operatorname{Im}(\alpha F)(\zeta(t))}{z-\zeta(t)} d t
\end{aligned}
$$

where

$$
G(z)=\frac{1}{2 \pi i} \int_{\overline{C A}} \frac{\alpha F(\zeta)}{z-\zeta} d \zeta
$$

in the last equation we used the relation $\alpha F=\operatorname{Re}(\alpha F)+i \operatorname{Im}(\alpha F)$. For $-1<$ $s<0$ we clearly have

$$
\lim _{\substack{z \zeta(s)+i \alpha \varepsilon \\ \varepsilon \rightarrow 0+}} \int_{0}^{1} \frac{\alpha i \operatorname{Im}(\beta F)(\zeta(t))}{z-\zeta(t)} d t=\int_{0}^{1} \frac{\alpha i \operatorname{Im}(\beta F)(\zeta(t))}{\zeta(s)-\zeta(t)} d t
$$

and, similarly to $(10.27)$,

$$
\lim _{\substack{z(s)+i \alpha \varepsilon \\ \varepsilon \rightarrow 0+}} \int_{-1}^{0} \frac{\alpha i \operatorname{Im}(\alpha F)(\zeta(t))}{z-\zeta(t)} d t=\pi \operatorname{Im}(\alpha F)(\zeta(s))+i \int_{-1}^{0} \frac{\operatorname{Im}(\alpha F)(\zeta(t))}{s-t} d t .
$$

Substituting (10.30) into (10.29) and using the last two relations, we obtain (10.31)

$$
\begin{aligned}
\pi^{2} \widetilde{H}^{2} u(s)=\operatorname{Re}\{-2 \pi i \alpha[F(\zeta(s))+G(\zeta(s))]-\pi \operatorname{Im}(\alpha F)(\zeta(s)) \\
\left.-i \int_{-1}^{0} \frac{\operatorname{Im}(\alpha F)(\zeta(t))}{s-t} d t-\alpha \int_{0}^{1} \frac{i \operatorname{Im}(\beta F)(\zeta(t))}{\zeta(s)-\zeta(t)} d t\right\} \\
=\pi \operatorname{Im}(\alpha F)(\zeta(s))+2 \pi \operatorname{Im}(\alpha G)(\zeta(s))-\operatorname{Re} \alpha \int_{0}^{1} \frac{i \operatorname{Im}(\beta F)(\zeta(t))}{\zeta(s)-\zeta(t)} d t
\end{aligned}
$$


Now, when $-1<s<0$,

$$
\alpha F(\zeta(s))=\lim _{\varepsilon \rightarrow 0+} \int_{-1}^{0} \frac{\alpha u(t)}{\alpha s+i \alpha \varepsilon-\alpha t} d t+\int_{0}^{1} \frac{\alpha u(t)}{\alpha s-\beta t} d t,
$$

and using (10.27) we get

$$
\alpha F(\zeta(s))=-i \pi u(s)+\int_{-1}^{0} \frac{u(t)}{s-t} d t+\int_{0}^{1} \frac{u(t)}{s-\beta t / \alpha} d t .
$$

Similarly, when $0<s<1$,

$$
\begin{aligned}
\beta F(\zeta(s)) & =\lim _{\varepsilon \rightarrow 0+} \int_{-1}^{0} \frac{\beta u(t)}{\beta s+i \beta \varepsilon-\alpha t} d t+\int_{0}^{1} \frac{\beta u(t)}{\beta s-\beta t} \\
& =-i \pi u(s)+\int_{0}^{1} \frac{u(t)}{s-t} d t+\int_{-1}^{0} \frac{u(t)}{s-\alpha t / \beta} d t .
\end{aligned}
$$

Using (10.32), (10.33) in (10.31), we get $(10.34)$

$$
\begin{aligned}
\pi^{2} \widetilde{H}^{2} u(s)= & \pi\left[-\pi u(s)+\operatorname{Im} \int_{0}^{1} \frac{u(t)}{s-\beta t / \alpha} d t\right]+2 \pi \operatorname{Im}(\alpha G)(\zeta(s)) \\
& -\operatorname{Re}\left\{\alpha i \int_{0}^{1} \frac{1}{\alpha s-\beta t}\left[-\pi u(t)+\operatorname{Im} \int_{-1}^{0} \frac{u(\tau)}{t-\alpha \tau / \beta} d \tau\right]\right\} d t .
\end{aligned}
$$

Noting the cancellation

$$
\operatorname{Re} \int_{0}^{1} \frac{\alpha i}{\alpha s-\beta t} \pi u(t) d t+\pi \operatorname{Im} \int_{0}^{1} \frac{u(t)}{s-\beta \tau / \alpha} d t=0
$$

we get from (10.34)

$$
\begin{aligned}
\pi^{2} \widetilde{H}^{2} u(s)= & -\pi^{2} u(s)+2 \pi \operatorname{Im}(\alpha G)(\zeta(s)) \\
& +\int_{0}^{1} \operatorname{Im}\left(\frac{1}{s-\beta t / \alpha}\right) \int_{-1}^{0}\left(\operatorname{Im} \frac{1}{t-\alpha \tau / \beta}\right) u(\tau) d \tau d t
\end{aligned}
$$

provided $-1<s<0$.

Similarly, if $0<s<1$,

$$
\begin{aligned}
\pi^{2} \widetilde{H}^{2} u(s)= & -\pi^{2} u(s)+2 \pi \operatorname{Im}\left(\beta G_{1}\right)(\zeta(s)) \\
& +\int_{-1}^{0} \operatorname{Im}\left(\frac{1}{s-\alpha t / \beta}\right) \int_{-1}^{0}\left(\operatorname{Im} \frac{1}{t-\beta \tau / \alpha}\right) u(\tau) d \tau d t
\end{aligned}
$$

with a corresponding function $G_{1}$.

Noting that

$$
\operatorname{Im} \frac{1}{s-\beta t / \alpha} \operatorname{Im} \frac{1}{t-\alpha \tau / \beta}=-\sin ^{2}(\theta-\varphi) \frac{t}{s^{2}+t^{2}} \frac{\tau}{t^{2}+\tau^{2}}
$$

if $s t<0, t \tau<0$, we can rewrite (10.35), (10.36) in the form

$$
\pi^{2} \widetilde{H}^{2} u=-\pi^{2} u+\widetilde{T} u+\widetilde{D} u,
$$


where

$$
\widetilde{D} u(s)=\left\{\begin{array}{l}
2 \pi \operatorname{Im}(\alpha G)(\zeta(s)), \\
2 \pi \operatorname{Im}\left(\beta G_{1}\right)(\zeta(s)),
\end{array}\right.
$$

$$
\begin{gathered}
\tilde{T} u(s)=-\left\{\int_{-1}^{1} h(s, t)\left(\int_{-1}^{1} h(t, \tau) u(\tau) d \tau\right) d t\right\} \sin ^{2}(\theta-\varphi)=-K^{2} u(s), \\
K u(s)=\left[\int_{-1}^{1} h(s, t) u(t) d t\right] \sin (\theta-\varphi),
\end{gathered}
$$

and

$$
h(s, t)= \begin{cases}\frac{t}{s^{2}+t^{2}} & \text { if } s t<0, \\ 0 & \text { if } s t>0 .\end{cases}
$$

In the definition of $G(z)$, the denominator is uniformly positive in absolute value for all $z=\zeta(s)$, when $s$ varies in the interval

$$
s_{i-1}+\delta \leq s \leq s_{i+1}-\delta \text {, i.e., } \quad-3 / 4 \leq s \leq 3 / 4 \text {. }
$$

A similar remark applies to $G_{1}$. Hence the operator $\widetilde{D}$ is compact from $L_{0}^{1}$ to $L^{2}\left(s_{i-1}+\delta, s_{i+1}-\delta\right)$. The restriction of $\widetilde{D} u$ from $L_{0}^{1}$ to $L^{2}(\Delta)$, where $\Delta=\left(0, l_{0}\right) \backslash\left(s_{i-1}+\delta, s_{i+1}-\delta\right)$, is also compact. Indeed, on $\Delta, \widetilde{D} u$ coincides with $\pi^{2} \widetilde{H}^{2} u$, i.e., $\tilde{D} u=\pi^{2} \tilde{H}(\tilde{H} \tilde{u})$, and $\tilde{H} \tilde{u}$ is smooth in $\Delta$ (i.e. analytic). We write $\widetilde{H} u=\sum \chi_{j}(\widetilde{H} u)$, apply $\widetilde{H}$ to each $\chi_{j}(\widetilde{H} u)$, and express $\widetilde{H}\left(\chi_{j} \widetilde{H} u\right)$ in $\left(s_{j-1}-\delta, s_{j+1}+\delta\right)$ as in (10.37). We then find that $\tilde{H}^{2} u$ is a compact operator from $L_{0}^{1}$ into $L^{2}(\Delta)$

We have thus proved that $\widetilde{D}$, in $(10.37)$, is a compact operator from $L_{0}^{1}$ into $L^{2}\left(0, l_{0}\right)$.

Next,

$$
\begin{aligned}
\|K u\|_{L^{1}} & \leq \int_{-1}^{1} d s\left|\int_{-1}^{1} h(s, t) u(t) d t\right| \\
& \leq \int_{-1}^{1}\left(\int_{-1}^{1}|h(s, t)| d s\right)|u(t)| d t \leq \frac{\pi}{2} \int_{-1}^{1}|u(t)| d t
\end{aligned}
$$

which establishes (10.22). To prove (10.23) take first $-1<s<0$. Then

$$
\begin{aligned}
|K u(s)| & \leq \int_{0}^{1}|u(t)| \frac{t}{s^{2}+t^{2}} d t \leq c_{0} \int_{0}^{1} \frac{|u(t)|}{t-s} d t \\
& =c_{0} \int_{-\infty}^{\infty} \frac{1}{t-s}\left(\mid\left(u(t) \mid \chi_{[0,1]}\right) d t\right.
\end{aligned}
$$

where $c_{0}$ is such that

$$
\frac{t}{s^{2}+t^{2}} \leq \frac{c_{0}}{t-s}, \quad \text { i.e., } \quad c_{0}=\frac{1+\sqrt{2}}{2}
$$


Then

$$
\int_{-1}^{0}|K u(s)|^{2} d s \leq c_{0}^{2} \pi^{2} \int_{-1}^{0}\left(H\left(u \chi_{[0,1]}\right)\right)^{2} d t \leq c_{0}^{2} \pi^{2} \int_{-\infty}^{\infty}\left(u \chi_{[0,1]}\right)^{2} d t .
$$

A similar estimate holds for $0<s<1$ and thus

$$
\int_{-1}^{1}|K u(s)|^{2} d s \leq \frac{3+2 \sqrt{2}}{4} \pi^{2} \int_{-1}^{1}|u(t)|^{2} d t
$$

and (10.23) follows.

Proof of Lemma 10.6. As before it is sufficient to concentrate on $\widetilde{H}^{2}$ with $u$ restricted to the space $L_{0}^{p}$ and $\widetilde{H}^{2}$ considered in $L^{p}\left(0, l_{0}\right)$. Using the notation (10.24), we introduce a curve $\tilde{l}(t)$ consisting of the two tangents to $\vec{l}(t)$ at $t=s_{i}=0$. We write $l(t)=\vec{l}(t)$ and denote by $\widetilde{H}_{l}, \widetilde{H}_{\tilde{l}}$ the operators $\widetilde{H}$ defined with respect to $l$ and $\tilde{l}$, respectively. Set

$$
\widetilde{D}=\widetilde{H}_{l}-\widetilde{H}_{\tilde{l}} \text {. }
$$

If we can prove that $\widetilde{D}$ is a compact operator from $L_{0}^{1}$ into $L^{2}$, then the assertion of Lemma 10.6 will follow from Lemma 10.7. To prove that $\widetilde{D}$ is compact, introduce the function

$$
k_{l}(s, t)=\frac{\vec{\tau} \cdot(l(s)-l(t))}{|l(s)-l(t)|^{2}}
$$

and similarly $k_{\tilde{l}}$.

Let $-1<s<0$. If $-1<t<0$, then

$$
k_{i}(s, t)=\frac{1}{s-t},
$$

whereas

$$
\begin{aligned}
k_{l}(s, t) & =\frac{\vec{\tau}(s) \cdot\left[\vec{\tau}(s)(s-t)+d_{1}(s-t)^{2}\right]}{\left|\vec{\tau}(s)(s-t)+d_{1}(s-t)^{2}\right|^{2}} \\
& =\frac{1}{s-t}+d_{2}=k_{i}(s, t)+d_{2},
\end{aligned}
$$

where the $d_{j}$ 's denote uniformly continuous functions of $(s, t)$. On the other hand, if $0<t<1$, then

and

$$
k_{\tilde{l}}=\frac{\vec{\alpha} \cdot(\vec{\alpha} s-\vec{\beta} t)}{|\vec{\alpha} s-\vec{\beta} t|^{2}}
$$

$$
k_{l}=\frac{\left(\vec{\alpha}+d_{3} s\right) \cdot\left(\vec{\alpha} s-\vec{\beta} t+d_{3} s^{2}+d_{4} t^{2}\right)}{|\vec{\alpha} s-\vec{\beta} t|^{2}+d_{5}}=\frac{\vec{\alpha} \cdot(\vec{\alpha} s-\vec{\beta} t)+d_{6}}{|\vec{\alpha} s-\vec{\beta} t|^{2}+d_{5}},
$$

where $\left|d_{5}\right| \leq C\left(|s|^{3}+s^{2} t+|s| t^{2}+t^{3}\right),\left|d_{6}\right| \leq C\left(t^{2}+t|s|+s^{2}\right)$. Since $|\vec{\alpha} s-\vec{\beta} t|^{2} \geq$ $c\left(s^{2}+t^{2}\right), c>0$, we deduce that

$$
k_{l}=k_{l}+d_{7}, \quad d_{7} \text { continuous. }
$$


From (10.39), (10.40) we see that, for $-1<s<0$,

$$
(\widetilde{D} u)(s)=\int_{-1}^{1} d(s, t) u(t) d t,
$$

where $d$ is a continuous function. The same result can be proved for $0<s<1$ Consequently $\widetilde{D}$ is a compact linear operator from $L_{0}^{p}$ into $L_{0}^{p}$.

Set

$$
\lambda=\frac{\left(\varepsilon_{1}-\varepsilon_{2}\right)^{2}}{4 \varepsilon_{1} \varepsilon_{2}} .
$$

We return to equations (10.18). Using Lemma 10.6 we can rewrite the second equation in the form

$$
[(1+\lambda) E-\lambda T] J+D J+\widetilde{T}_{2}(I, J)=-\widetilde{J}_{0},
$$

where $E$ is the identity operator. Set

$$
B=[(1+\lambda) E-\lambda T]^{-1}=(1+\lambda)^{-1} \sum_{n=0}^{\infty}\left(\frac{\lambda}{1+\lambda}\right)^{n} T^{n} ;
$$

this is a bounded linear operator in $X_{p}$ provided

$$
\|T\|_{L^{p}}<\left|\frac{1+\lambda}{\lambda}\right| \text {. }
$$

When this condition is satisfied we can rewrite (1.18) in the form

$$
\begin{gathered}
I+\widetilde{T}_{1}(I, J)=-I_{0}, \\
J+B D+B \widetilde{T}_{2}(I, J)=-B \widetilde{J}_{0},
\end{gathered}
$$

which looks like a Fredholm system.

By Lemma 10.6, (10.44) is satisfied if

$$
\begin{gathered}
\frac{1}{4}<\left|\frac{1+\lambda}{\lambda}\right|, \quad p=1, \\
\frac{3+2 \sqrt{2}}{4}<\left|\frac{1+\lambda}{\lambda}\right|, \quad p=2 .
\end{gathered}
$$

Recalling that $\varepsilon_{j}=\varepsilon_{j}^{\prime}+i \varepsilon_{j}^{\prime \prime}, \quad \varepsilon_{j}^{\prime}>0, \varepsilon_{j}^{\prime \prime} \geq 0$, we have

$$
\left|\frac{1+\lambda}{\lambda}\right|=\left|\frac{\left(\varepsilon_{1}+\varepsilon_{2}\right)^{2}}{\left(\varepsilon_{1}-\varepsilon_{2}\right)^{2}}\right|=\frac{\left(\varepsilon_{1}^{\prime}+\varepsilon_{2}^{\prime}\right)^{2}+\left(\varepsilon_{1}^{\prime \prime}+\varepsilon_{2}^{\prime \prime}\right)^{2}}{\left(\varepsilon_{1}^{\prime}-\varepsilon_{2}^{\prime}\right)^{2}+\left(\varepsilon_{1}^{\prime \prime}-\varepsilon_{2}^{\prime \prime}\right)^{2}}>1,
$$

so that $(10.46)$ is always satisfied. Consequently we prefer to work in the space $X_{1}$. However this requires a careful look at all the terms in $\widetilde{T}_{2}$, ensuring that they each are a compact operator in $X_{1}$. Recall that in the operator $\widetilde{T}_{2}$ there enter operators which are obtained when we substitute $I$ from (10.15) into (10.16). The "worst" operator in $\widetilde{T}_{2}$ is

$$
c_{1} f_{1}^{\prime}(s) \int_{0}^{l_{0}}(\widetilde{H} u)(t)\left(\frac{\Psi_{2}}{\varepsilon_{2}}-\frac{\Psi_{1}}{\varepsilon_{1}}\right)(x(s)-y(t)) d t,
$$


$c_{1}$ constant. If $u \in L^{1}$ then $\widetilde{H} u$ is in $\left(L^{1}\right)_{\text {weak }}$, so that in order to make sense out of the above operator we must write it in the form

$$
c_{2} f_{1}^{\prime}(s) \int u\left(t^{\prime}\right) d t^{\prime} \int \frac{\vec{\tau}(t) \cdot\left(\vec{l}(t)-\vec{l}\left(t^{\prime}\right)\right)}{\left|\vec{l}(t)-\vec{l}\left(t^{\prime}\right)\right|^{2}} \log \frac{1}{|x(s)-y(t)|} d t+T_{0} u,
$$

$c_{2}$ is another constant, and $T_{0}$ is a compact operator in $X_{1}$. The inner integral has the form

$$
K\left(t^{\prime}, s\right)=\widetilde{H}\left(\log \frac{1}{|x(s)-y(t)|}\right)\left(t^{\prime}\right) .
$$

As in the proof of the previous two lemmas, it is enough to consider what happens in a neighborhood of $s_{i}=0$ when $\vec{l}(t)$ consist of two line segments meeting at $t=0$. Using the same notation as in Lemma 10.7, this reduces to studying the operator $K$ :

$$
K u(s)=\int_{-1}^{1} \log (s-\tau)\left(T_{0} u\right)(\tau) d \tau,
$$

where

$$
T_{j} u(s)=\int_{-1}^{1} h_{j}(s, t) u(t) d t
$$

and

$$
h_{0}(s, t)= \begin{cases}\frac{1}{s-t} & \text { if } s t>0 \\ \frac{s}{s^{2}+t^{2}} & \text { if } s t<0 .\end{cases}
$$

Later on we shall also need to examine $T_{1} u$ where

$$
h_{1}(s, t)= \begin{cases}\frac{t}{s^{2}+t^{2}} & \text { if } s t<0, \\ 0 & \text { if } s t>0 .\end{cases}
$$

The operator $K$ does not appear to be compact or even bounded if we consider it in the $L^{1}$-norm. We shall therefore resort to a weighted $L^{1}$-norm

$$
\|u\|_{\alpha}=\int_{-1}^{1} \frac{|u(t)|}{|t|^{\alpha}} d t, \quad 0<\alpha<1 .
$$

Lemma 10.8. The operator $u \rightarrow K u$ from $L_{0}^{1}$ into $L^{1}$ is a compact operator provided both $u$ and $K u$ are taken with the \|\|$_{\alpha}$ norm.

Here $L^{1}=L^{1}(-1,1)$ and $L_{0}^{1}=\left\{u \in L^{1} ; u(t)=0\right.$ if $\left.|t|>\frac{3}{4}\right\}$.

Proof. Write $v=K u$ and set

$$
\tilde{u}(t)=t^{-\alpha} u(t), \quad \tilde{v}(s)=s^{-\alpha} v(s) .
$$

Then

$$
\tilde{v}(s)=\int_{-1}^{1} \tilde{k}(s, t) \tilde{u}(t) d t \equiv(\widetilde{K} \tilde{u})(s)
$$


where

$$
\tilde{k}(s, t)=\frac{t^{\alpha}}{s^{\alpha}} \int_{-1}^{1} \log |s-\tau| h_{0}(\tau, t) d \tau,
$$

and it suffices to show that $\tilde{K}$ is compact from $L_{0}^{1}$ into $L^{1}$.

If $s>0$, then, for $t>0$,

$$
\tilde{k}(s, t)=\frac{t^{\alpha}}{s^{\alpha}}\left[\text { p.v. } \int_{0}^{1} \log |s-\tau| \frac{d \tau}{\tau-t}+\int_{-1}^{0} \log |s-\tau| \frac{\tau}{t^{2}+\tau^{2}} d \tau\right],
$$

whereas for $t<0$

$$
\tilde{k}(s, t)=\frac{t^{\alpha}}{s^{\alpha}}\left[\text { p.v. } \int_{-1}^{0} \log |s-\tau| \frac{d \tau}{\tau-t}+\int_{0}^{1} \log |s-\tau| \frac{\tau}{t^{2}+\tau^{2}} d \tau\right] .
$$

A similar representation holds for $s<0$. Using properties of the Hilbert transform it is easily seen that

$$
\begin{aligned}
& \tilde{k}(s, t) \text { is a continuous function of }(s, t) \text { for all }(s, t) \text { except } \\
& \text { possibly } s=0, t=0 \text { or } s=t .
\end{aligned}
$$

If we can prove that

$$
|\tilde{k}(s, t)| \leq \frac{C t^{\alpha}}{s^{\alpha}}\left[(\log |t|)^{2}+|\log | s|\log | t \mid\right]
$$

then

$$
|\tilde{k}(s, t)| \leq \frac{C}{s^{\alpha}}|\log | s|| .
$$

Using this estimate and (10.53), we can deduce by a standard argument that $\widetilde{K}$ is a compact operator from $L_{0}^{1}$ into $L^{1}$.

To prove (10.54) it suffices to consider the case $s>0, t>0$. Then

$$
\begin{aligned}
\tilde{k}(s, t) & =\frac{t^{\alpha}}{s^{\alpha}}\left[\mathrm{p} . \mathrm{v} \cdot \int_{0}^{1} \log |s-\tau| \frac{d \tau}{\tau-t}+\int_{-1}^{0} \log |s-\tau| \frac{\tau}{t^{2}+\tau^{2}} d \tau\right] \\
& \equiv \frac{t^{\alpha}}{s^{\alpha}}\left(J_{1}+J_{2}\right)
\end{aligned}
$$

and

$$
\begin{aligned}
\left|J_{2}\right| & \leq\left|\int_{|\tau|<s / 2}+\int_{s / 2<|\tau|<2 s}+\int_{|\tau|>2 s \mid}\right| \\
& \leq C|\log s||\log t|+\frac{C}{s}\left|\int_{-s / 2}^{2 s} \log \right| s-\tau|d \tau|+C|\log s||\log t| \\
& \leq C|\log s||\log t| .
\end{aligned}
$$

Next

$$
J_{1}=\text { p.v. } \int_{0}^{2 t} \log |s-\tau| \frac{d \tau}{\tau-t}+\int_{2 t}^{1} \log |s-\tau| \frac{d \tau}{\tau-t} \equiv I_{1}+I_{2}
$$


Substituting $\tau=t+\eta$ in $I_{1}$ we get

$$
I_{1}=\text { p.v. } \int_{-t}^{t} \frac{\log |s-t-\eta|}{\eta} d \eta=\int_{0}^{t} \frac{\log |s-t-\eta|-\log |s-t+\eta|}{\eta} d \eta
$$

so that, upon substituting $\eta=|s-t| \xi$,

$$
\left|I_{1}\right| \leq\left|\int_{0}^{\infty} \frac{\log |(1+\xi) /(1-\xi)|}{\xi} d \xi\right| \leq C .
$$

To estimate $I_{2}$ we substitute $\tau=2 t+\eta t$ to get

$$
I_{2}=\int_{0}^{1 / t-2} \frac{\log |s-2 t-\eta t|}{\eta+1} d \eta
$$

we may assume that $1 / t>2$. Writing

$$
s-2 t-\eta t=t(\gamma-\eta) \quad \text { where } \gamma=\frac{s-2 t}{t},
$$

we obtain

$$
\begin{aligned}
\left|I_{2}\right| & \leq \int_{0}^{1 / t-2} \frac{|\log t|}{\eta+1} d \eta+\left|\int_{0}^{1 / t-2} \frac{\log |\eta-\gamma|}{\eta+1} d \eta\right| \\
& \leq|\log t| \log \frac{1}{t}+\tilde{I}_{2}
\end{aligned}
$$

where

$$
\tilde{I}_{2}=\left(\int_{\{|\eta-\gamma|<1\}}^{1 / t-2}+\int_{\{\eta<\gamma-1\}}^{1 / t-2}+\int_{\{\eta>\gamma+1\}}^{1 / t-2} \begin{array}{l}
0 \\
0
\end{array}\right) \frac{|\log | \eta-\gamma||}{\eta+1} d \eta .
$$

The first term in $\tilde{I}_{2}$ is bounded by

$$
2 \int_{0}^{1}|\log \xi| d \xi
$$

and each of the remaining two terms is bounded by

$$
\log \frac{1}{t} \int_{0}^{1 / t} \frac{d \eta}{\eta+1}=(\log t)^{2} .
$$

It follows that $\left|I_{2}\right| \leq C(\log t)^{2}$. Combining this with (10.58), (10.57), we see that

$$
\left|J_{1}\right| \leq C(\log t)^{2} .
$$

Recalling (10.56), (10.55), the assertion (10.54) follows.

Having proved Lemma 10.8, we remark that the compactness of all the other operators in $\tilde{T}_{2}$ is proved much more easily. However, since we shall be using the $\alpha$-norm \|\|$_{\alpha}$, we still must extend also Lemma 10.6 and the estimate (10.22).

The proof of Lemma 10.6, for the $\alpha$-norm, remains essentially the same; the only difference is that now we have a slightly different estimate on $T_{1}$, namely

$$
\left\|T_{1} u\right\|_{\alpha} \leq \frac{\pi}{2 \cos (\pi \alpha / 2)}\|u\|_{\alpha}
$$


$T_{1}$ is defined by (10.50), (10.52). The proof of this estimate follows from

$$
\begin{aligned}
\left\|T_{1} u\right\|_{\alpha} & \leq\|u\|_{\alpha} \sup _{t} \int_{-1}^{1} \frac{\left|h_{1}(s, t)\right|}{|s|^{\alpha}}|t|^{\alpha} d s \\
& \leq \int_{0}^{\infty} \frac{d \eta}{\eta^{\alpha}\left(1+\eta^{2}\right)} \quad(s=t \eta) \\
& =\frac{\pi}{2 \cos (\pi \alpha / 2)} .
\end{aligned}
$$

From (10.59) we conclude that instead of (10.22) the following holds:

$$
\|T\|_{\alpha} \leq \frac{1}{4 \cos ^{2}(\pi \alpha / 2)}\|u\|_{\alpha} .
$$

Denote by $X_{1, \alpha}$ the space $X_{1}$ where the $L^{1}$-norm is taken with weight $\omega(t)$,

$$
\omega(t)= \begin{cases}\left|t-s_{i}\right|^{-\alpha} & \text { if }\left|t-s_{i}\right|<\delta \\ 1 & \text { if }\left|t-s_{i}\right|>\delta\end{cases}
$$

for some small $\delta>0$.

Notice that working with the space $X_{1, \alpha}$, the condition (10.44) becomes

$$
\|T\|_{\alpha} \equiv\|T\|_{X_{1, \alpha}}<\left|\frac{1+\lambda}{\lambda}\right|
$$

and, in view of $(10.60),(10.47)$, this condition is always satisfied if $4 \cos ^{2}(\pi \alpha / 2)$ $\geq 1$, i.e., if $0<\alpha \leq 2 / 3$. In this case, then, the system (10.18) is of Fredholm type.

We summarize:

Theorem 10.9. The system (10.18) in $X_{1, \alpha}(\alpha \in(0,2 / 3])$ is equivalent to the Fredholm system (10.45) where $B D$ and $B \widetilde{T}_{2}$ are compact operators in $X_{1, \alpha}$. Acknowledgment. We would like to thank Dr. Allen Cox from Honeywell for suggesting the problem studied in this paper.

\section{REFERENCES}

1. A. Bellout and A. Friedman, Scattering by strip grating, J. Math. Anal. Appl. (to appear).

2. A. Benaldi, Numerical analysis of the exterior boundary value problem for the time-harmonic Maxwell equations by a boundary finite element method. Part 1: The continuous problem; Part 2: The discrete problem, Math. Comp. 167 (1984), 29-46, 47-68.

3. N. G. De Bruijn, Asymptotic methods in analysis, North-Holland, Amsterdam, 1958.

4. C. A. Coulson and A. Jeffrey, Waves, 2nd ed., Longman, London, 1977.

5. T. K. Gaylord and M. G. Moharam, Analysis and applications of optimal diffraction by gratings, Proc. IEEE 73 (1985), 894-937.

6. I. S. Gradsteyn and I. M. Ryzhik, Tables of integrals, series and products, Academic Press, New York, 1965.

7. C. Muller, Foundations of mathematical theory of electromagnetic waves, Springer-Verlag, Berlin, 1969. 
8. J. C. Nedelec and F. Starling, Integral equation methods in quasi periodic diffraction problem for the time harmonic Maxwell's equations, Ecole Polytechnique, Centre de Mathematiques Appliquees, Tech. Report \#756, April 1988.

9. R. Petit (Editor), Electromagnetic theory of gratings, Topics in Current Phys., vol. 22, Springer-Verlag, Heidelberg, 1980.

Department of Mathematics, University of Minnesota, Minneapolis, Minnesota 55455

Institute for Mathematics and its Applications, University of Minnesota, MinNeapoLIS, MiNNESOTA 55455 